\title{
Du Fondo Barbatelli à la Porte du Vésuve : Une fenêtre d'étude dans le faubourg septentrional de Pompéi
}

Campagne 2020

\section{Bastien Lemaire et Jean-Pierre Brun}

\section{OpenEdition}

\section{Journals}

Édition électronique

URL : https://journals.openedition.org/baefe/2378

DOI : $10.4000 /$ baefe. 2378

ISSN : 2732-687X

Éditeur

ResEFE

Référence électronique

Bastien Lemaire, Jean-Pierre Brun, «Du Fondo Barbatelli à la Porte du Vésuve : Une fenêtre d'étude dans le faubourg septentrional de Pompéi » [notice archéologique], Bulletin archéologique des Écoles françaises à l'étranger [En ligne], Italie, mis en ligne le 22 mai 2021, consulté le 24 mai 2021. URL http://journals.openedition.org/baefe/2378; DOI : https://doi.org/10.4000/baefe.2378

Ce document a été généré automatiquement le 24 mai 2021.

\section{(c) (i) $\odot$}

Le Bulletin archéologique des Écoles françaises à l'étranger est mise à disposition selon les termes de la Licence Creative Commons Attribution - Pas d'Utilisation Commerciale - Pas de Modification 4.0 International. 


\title{
Du Fondo Barbatelli à la Porte du Vésuve : Une fenêtre d'étude dans le faubourg septentrional de Pompéi
}

Campagne 2020

\author{
Bastien Lemaire et Jean-Pierre Brun
}

\section{NOTE DE L'AUTEUR}

Date précise de l'opération : 12 octobre-31 octobre 2020

Autorité nationale présente : Parco archeologico di Pompéi - MiC

Numéro de mission : Concession de fouilles MIBACT DGABAP Prot. n. $0023242 \mathrm{du}$ 04/08/2020.

Composition de l'équipe de terrain : Le projet de recherche «Du Fondo Barbatelli à la Porte du Vésuve : une fenêtre d'étude dans le faubourg septentrional de Pompéi » est conduit sous la direction de Jean-Pierre Brun (Collège de France) et Bastien Lemaire (UMR 5140 ASM - Université Paul Valéry Montpellier 3). L'équipe de recherche est composée de Laetitia Cavassa (céramologue, Aix Marseille Université, CNRS, Centre Camille Jullian), Guilhem Chapelin, (architecte, CNRS, Centre Jean Bérard), Marina Covolan (archéologue, doctorante à l'Université de Salerne), Nicolas Leys (photogrammètre, Institut des Sciences du Calcul et des Données - Sorbonne Université) et Arnaud Watel (géomaticien, Éveha). Lors de cette campagne, l'équipe de fouille était également constituée de Simon Azema (archéologue, médiateur culturel), Florian Germain (archéologue, étudiant à l'Université de Liège), Noémie Ledouble (archéologue, médiatrice culturelle), Lucie Robert (anthropologue), François Sterna (archéologue) et Charlotte Villers (archéologue, étudiante à l'Université de Liège). Cette opération a été réalisée du 12 au 31 octobre 2020 grâce aux crédits du ministère de l'Europe et des Affaires étrangères (Mission archéologique «Italie du Sud ») et à une subvention de la Fondation du Collège de France, par le Centre Jean Bérard, le Collège de France et l'UMR 5140 ASM (CNRS - Université Paul Valéry Montpellier 3), en étroite 
collaboration avec le Parco archeologico di Pompei qui nous a permis de travailler dans les meilleures conditions.

Partenariats institutionnels : CJB, Collège de France, UMR 5140 ASM - Université Paul Valéry Montpellier 3, LabEx ARCHIMEDE

Établissements porteurs de l'opération : $\mathrm{CJB}$, Collège de France

Remerciements : Nous remercions tout particulièrement le professeur Massimo Osanna, directeur du Parc, la dott.ssa Marialaura Iadanza, fonctionnaire archéologue responsable de notre secteur, Vincenzo Sabini, assistant technique, ainsi que la dott.ssa Valeria Amoretti, fonctionnaire anthropologue, la dott.ssa Luana Toniolo et le dott. Domenico Busiello, responsables des dépôts. La fouille a été réalisée dans le cadre d'une concession accordée par le Ministero per i Beni e le Attività Culturali e del Turismo (Concession de fouilles DGABAP Prot. n. $0023242 \mathrm{du}$ 04/08/2020).

Nous souhaitons également remercier toute l'équipe du Centre Jean Bérard pour leur précieux soutien et tout particulièrement Claude Pouzadoux et Priscilla Munzi, directrice et directriceadjointe.

Cette campagne de fouille a fait notamment l'objet d'un enregistrement en ligne directement sur le terrain, sur la base de données Syslat et via le logiciel iSyslat, grâce à du matériel fourni par le laboratoire d'Archéologie des Sociétés Méditerranéennes du CNRS (UMR 5140) et le LabEx ARCHIMEDE.

Données scientifiques produites :

https://centrejeanberard.cnrs.fr/spip.php?article106\&lang=fr

Chroniques de l'EFR :

http://journals.openedition.org/cefr/4941

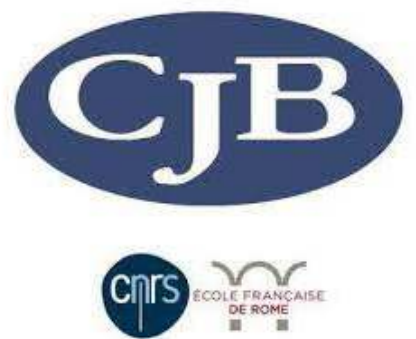

\section{Introduction}

1 Le faubourg septentrional de Pompéi, en avant de la porte du Vésuve, a été dégagé de manière discontinue à partir de l'extrême fin du $\mathrm{XIX}^{\mathrm{e}}$ siècle et la première décennie du siècle suivant.

2 En effet, bien que repérée dès 1811 , la porte du Vésuve fut dégagée de manière intermittente dès 1902, jusqu'aux fouilles de la zone directement au nord des remparts, entre 1907 et 1910. Plus au nord, une partie d'une villa suburbaine fut mise au jour dans le fondo Masucci d'Aquino entre 1897 et 1899. Ensuite, des fouilles furent réalisées directement au sud de cette parcelle, dans le fondo Barbatelli, au cours de deux campagnes: entre octobre 1899 et février 1900, puis entre septembre 1900 et 
février 1901. Enfin, de 1997 à 2002, un projet de recherche de l'Istituto Archeologico Germanico, mené sous la direction de Florian Seiler (DAI, Berlin) a intéressé la Regio VI, Insula 16 et la porte du Vésuve ${ }^{1}$, mais ne s'est que peu étendu extra muros. En effet, ce secteur périurbain n'a que peu retenu l'attention des chercheurs, en dehors de l'étude des monuments funéraires. Le programme de recherche actuel concerne la zone située directement au nord de la porte de la ville et s'étend sur plus de 130 mètres, permettant d'avoir un aperçu de l'espace périurbain septentrional ${ }^{2}$.

3 La réinterprétation des documents concernant les anciens dégagements à la lumière des récentes investigations de terrain autorisent à proposer de regrouper les vestiges des fondi Masucci d'Aquino et Barbatelli en une vaste villa suburbana à laquelle on accédait par une colonnade donnant sur la voie menant à la ville ${ }^{3}$. Notons que, lors de l'éruption du Vésuve en 79 apr. J.-C., la partie méridionale de cet ensemble résidentiel était occupée par un complexe artisanal multifonctionnel, présentant notamment le plus vaste atelier polymétallurgique connu à ce jour à Pompéi. Par ailleurs, à proximité de la porte du Vésuve, en plus des quatre monuments funéraires, le tracé de l'aqueduc menant au castellum aquae de la ville a été reconnu, mais présente des bifurcations en amont ${ }^{4}$ impliquant que des installations périurbaines étaient reliées à cet ouvrage sous contrôle de la cité. Deux espaces délimités par des murs bordent également la voie, mais leur fonction reste incertaine. Enfin, lors des dégagements du début du XXe siècle, il a été mis en évidence que la porte de la ville, détruite par le tremblement de terre de 62/63 apr.J.-C., n'était toujours pas reconstruite et que les tombeaux présents sur la zone étaient recouverts par des déchets et matériaux probablement issus des destructions liées à cette catastrophe et aux déblaiements réalisés dans le centre urbain.

4 Notre recherche est donc centrée sur le passage de l'urbain au périurbain, avec pour but de mieux comprendre cet espace d'interface entre la ville et sa campagne. Notre fenêtre d'étude permet en effet d'observer l'organisation et la gestion d'un secteur où s'articulent des structures défensives, viaires, hydrauliques, funéraires, résidentielles, artisanales et des décharges installées sur des terrains publics ou privés. Cherchant à mieux cerner le rôle et les fonctions des espaces périurbains dans l'histoire socioéconomique des cités antiques, ce projet s'intègre aux recherches récentes menées sur les périphéries urbaines des sociétés anciennes ; loin de se limiter au monde funéraire, il teste la nature des espaces suburbains et leur plurifonctionnalité, définissant ainsi la cité de manière plus précise et dans son intégralité.

\section{La campagne de fouille 2020}

5 Depuis 2019, les fouilles ont concerné l'espace directement en avant de la porte du Vésuve, lequel a été divisé en zones, puis en secteurs, pour des raisons pratiques d'enregistrement et de localisation des vestiges archéologiques encore visibles ou mis au jour. La campagne 2020 (fig. 1) a concerné l'espace directement en avant de la porte du Vésuve et a été l'occasion de fouiller deux espaces (secteurs 20 et 41) se situant de part et d'autre de la voie sortant de la ville en direction du nord. De plus, un relevé photogrammétrique précis a été réalisé sur une structure importante du secteur 10, dégagée l'année précédente. L'enregistrement des données de terrain s'est effectué à l'aide du système d'enregistrement archéologique Syslat Terminal et des relevés topographiques et photogrammétriques ont été réalisés afin d'obtenir des données 
planimétriques et les insérer dans un système d'information géographique (SIG). Un nord théorique a été utilisé afin de décrire les vestiges et correspond à l'axe viaire sortant de la ville et se dirigeant vers le Vésuve plus au nord.

Fig. 1. Plan général des vestiges en avant de la porte du Vésuve et de l'emprise des sondages de la campagne 2020.

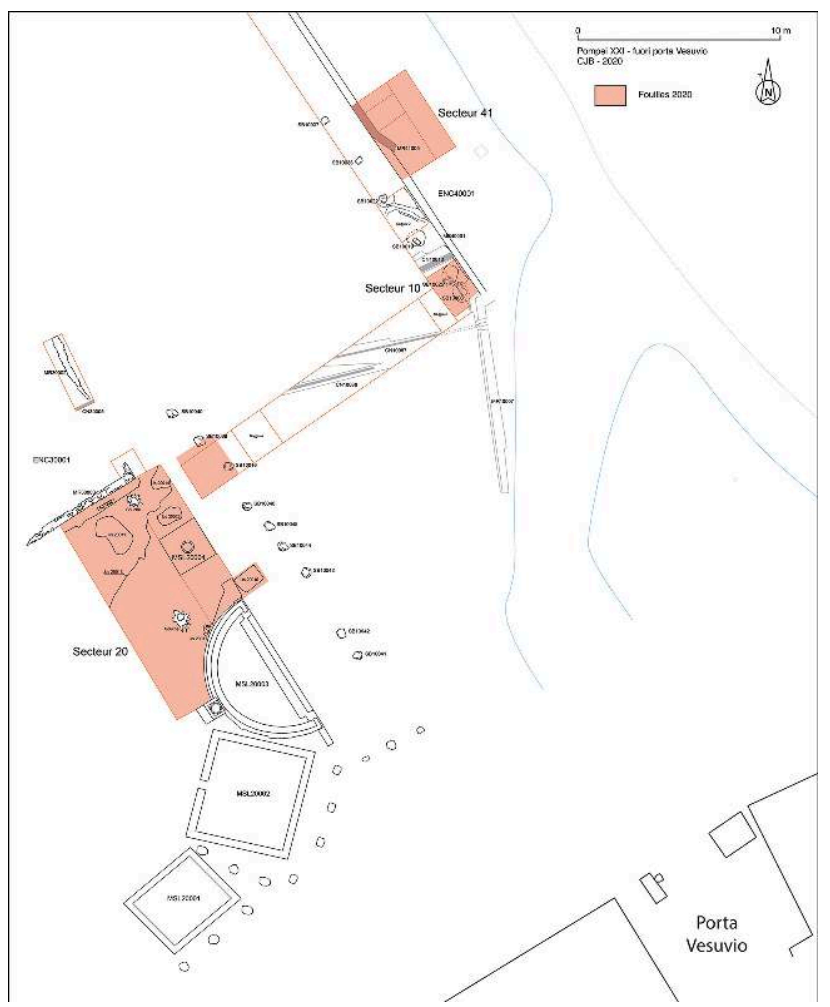

B. Lemaire, G. Chapelin, sur concession du MiC - PA Pompei. Tous droits réservés.

6 Bien que l'étude complète du matériel archéologique prélevé n'ait pu être menée à bien au terme de la campagne de fouille et qu'une mission d'étude soit prévue en 2021, les premiers résultats obtenus, remis dans le contexte de la zone d'étude autorisent déjà une première synthèse phasée des vestiges découverts. Ainsi, alors que des niveaux datant de la fin de la période républicaine et des premiers temps de la colonie romaine avaient été observés lors de la campagne précédente, la majorité des vestiges identifiés cette année remontent au $\mathrm{I}^{\mathrm{er}}$ siècle apr. J.-C., voire à la seconde moitié du $\mathrm{I}^{\mathrm{er}}$ siècle apr. J.-C. Durant cette période, après le tremblement de terre de 62/63 apr. J.-C., les opérations de restitutio du tribun Titus Suedius Clemens - de la fin du troisième quart $\mathrm{du} \mathrm{I}^{\mathrm{er}}$ siècle apr. J.-C. - ont pu être associées à des structures particulières, jusqu'aux niveaux liés à l'éruption du Vésuve de 79 apr. J.-C. Enfin, depuis les dégagements du début du $\mathrm{XX}^{\mathrm{e}}$ siècle, de nombreux remaniements et installations ayant impacté le site ont pu être identifiés.

\section{La zone 1 : secteur 10}

Les aménagements liés aux travaux du tribun T. Suedius Clemens

7 La zone 1 concerne la voie partant vers le nord, délimitée par les murs MR40001 et MR10007 à l'est, ainsi que par le mur MR30002 et les monuments funéraires MSL20003 
et MSL20004 à l'ouest. De nouveaux secteurs de fouille n'ont pas été ouverts dans cette zone, mais deux points sont venus compléter les données de la campagne 2019, sur des aménagements mis en relation avec les actions menées par le tribun Titus Suedius Clemens.

8 - À l'est de la voie et à l'extrémité orientale de la tranchée orientée est-ouest ouverte l'année précédente, une fenêtre d'environ $3,5 \mathrm{~m}^{2}$ a été rouverte autour de la borne SB10025 et de la stèle inscrite SB10008 attenante. L'objectif était de réaliser une photogrammétrie fine de cette dernière, portant l'inscription relative aux travaux menés par le tribun Titus Suedius Clemens et datant de la fin du troisième quart du $\mathrm{I}^{\mathrm{er}}$ siècle apr. J.-C. ${ }^{5}$.

9 La structure SB10008 ${ }^{6}$ est réalisée dans un bloc de calcaire dur blanc, régulier en surface et irrégulier dans la partie enterrée. Elle mesure au maximum $162 \mathrm{~cm}$ de haut, $65 \mathrm{~cm}$ du nord au sud pour $26 \mathrm{~cm}$ d'est en ouest et présente un ressaut allant jusqu'à $5 \mathrm{~cm}$ à la liaison entre la partie visible et la partie enterrée. La partie supérieure portant l'inscription mesure entre 70 et $72 \mathrm{~cm}$ de haut sur $60 \mathrm{~cm}$ du nord au sud pour $25,5 \mathrm{~cm}$ d'est en ouest. L'inscription est réalisée sur la face ouest de la stèle, visible depuis la voie et porte une inscription (entre $3,5 \mathrm{~cm}$ et $53 \mathrm{~cm}$ du haut de la stèle) de 8 lignes irrégulières (allant de 6,2 à $2,5 \mathrm{~cm}$ de hauteur).

10 Cette stèle inscrite SB10008 révèle les opérations de restitutio du tribun Titus Suedius Clemens, envoyé par l'empereur Vespasien, concernant des loca publica ${ }^{7}$ : c'est à dire la remise à la colonie des terrains publics illégalement occupés par des privés, possiblement à la suite du tremblement de terre de 62/63 apr. J.-C. ${ }^{8}$. Cette structure est installée dans une fosse (FS10030) mesurant jusqu'à $65 \mathrm{~cm}$ de diamètre et $80 \mathrm{~cm}$ de profondeur. Cette fosse étroite et profonde empêchant la réalisation d'une photographie de la stèle sur toute sa hauteur, il a été décidé de rouvrir cet espace afin de réaliser un modèle photogrammétrique (fig. 2). Celui-ci permet de tirer des vues et des coupes précises, qui viennent compléter les relevés effectués à la main et de l'intégrer à terme dans un modèle interactif. De plus, le texturage rendu possible par cette technique sera apte à révéler certaines traces de tailles et tracés préparatoires à l'inscription, lors d'un examen réalisé a posteriori en laboratoire. 
Fig. 2. La stèle inscrite SB10008 lors de la réalisation du modèle sur le terrain et sa vue de face tirée à partir de la photogrammétrie.

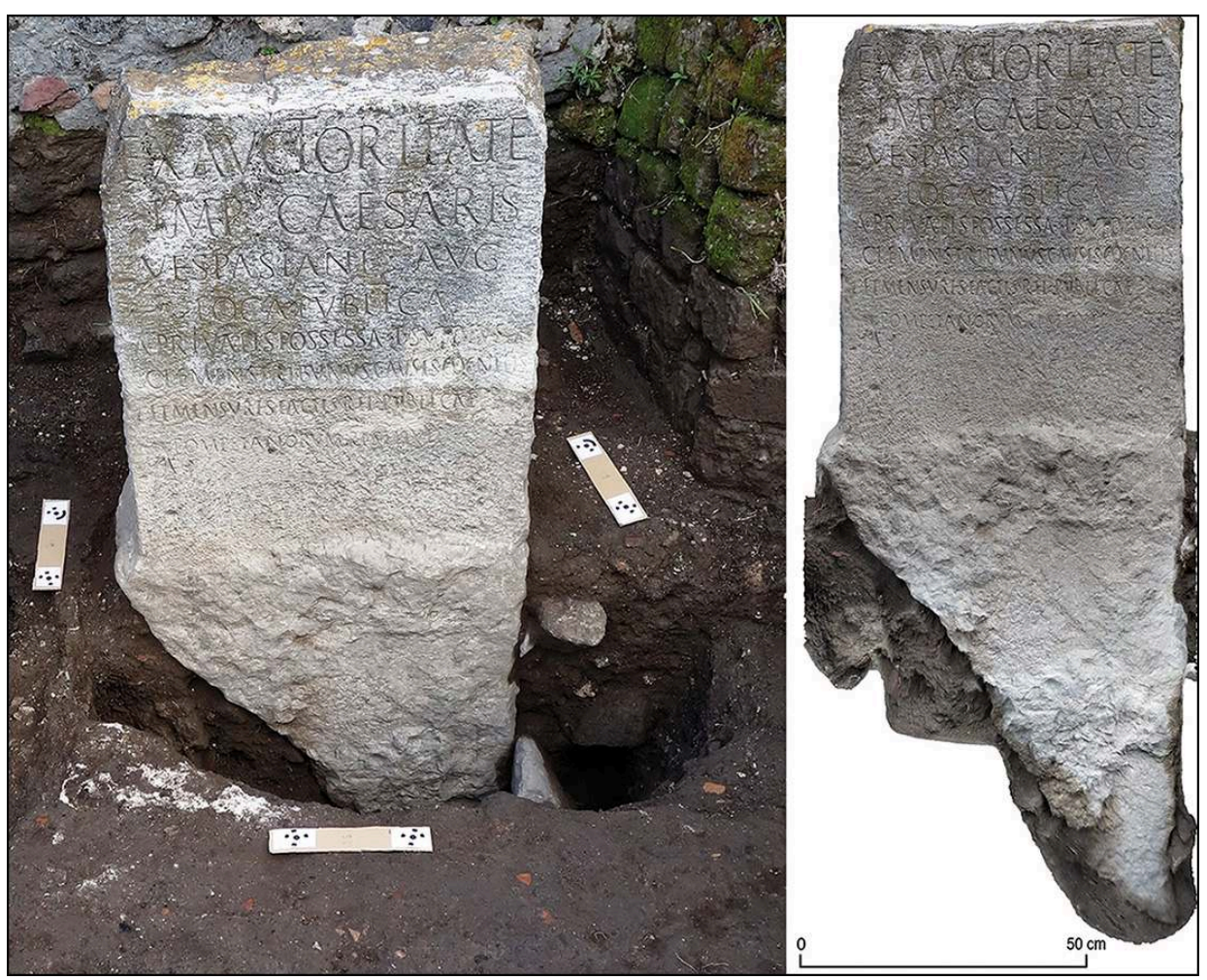

B. Lemaire, N. Leys, sur concession du MiC - PA Pompei. Tous droits réservés.

11 - À l'ouest de la voie et à l'extrémité occidentale de la tranchée orientée est-ouest, ouverte l'année précédente, le nettoyage des couches contemporaines a permis d'identifier les niveaux d'installation de la borne en trachyte SB10039. Cette dernière est contemporaine de la borne SB10016, fouillée l'année dernière et très probablement des autres bornes bordant la voie. La borne SB10039 est également installée dans une fosse (FS10095) creusée dans le sol SL10015 (fig. 3). La fosse FS10095 a été observée sur son tiers méridional, mesure $40 \mathrm{~cm}$ de diamètre (est-ouest) et a été partiellement fouillée sur $20 \mathrm{~cm}$ de profondeur. Son comblement (US 10096) est composé d'un limon brun foncé, relativement meuble et ne présentant que peu de matériel (céramique et faune). 
Fig. 3. La fosse d'installation FS10095 de la borne SB10039 (à droite) et la borne SB10016 (à gauche), vues de l'est.

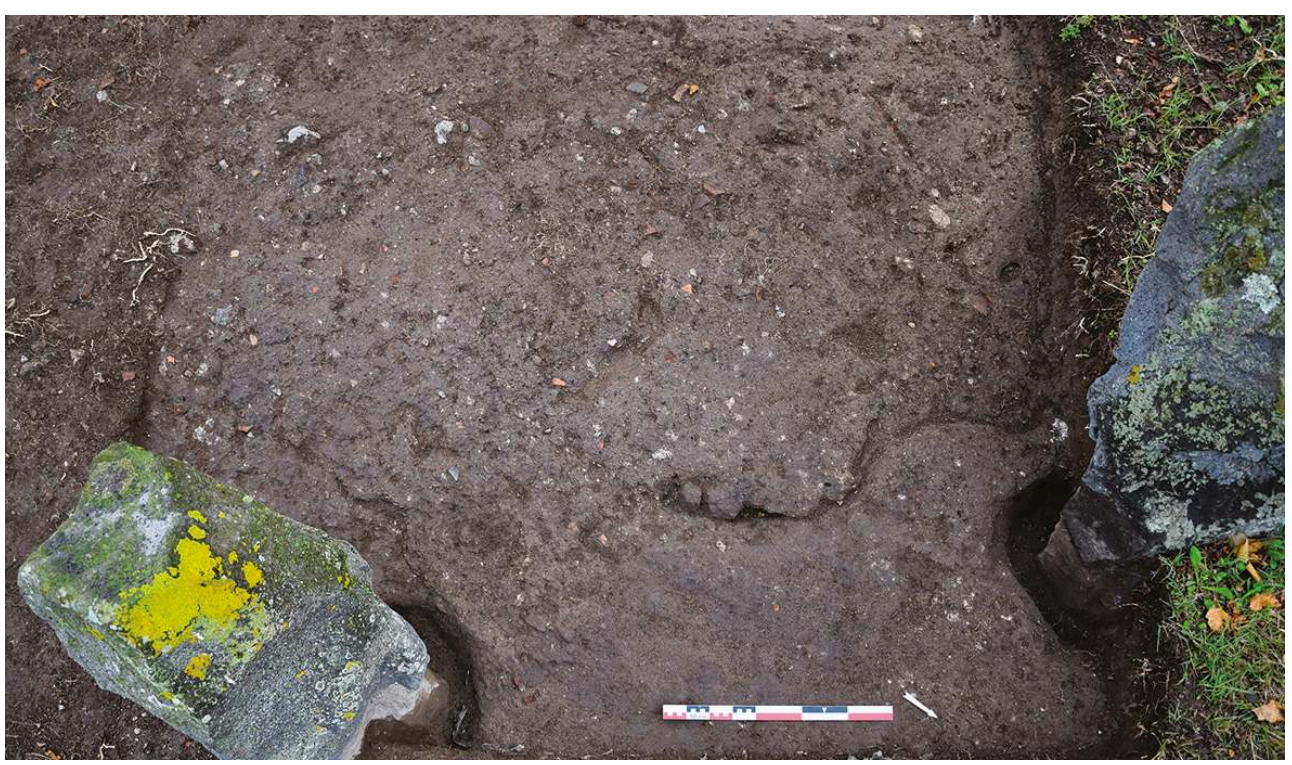

B. Lemaire, sur concession du MiC - PA Pompei. Tous droits réservés.

12 Aucun lien direct avec la stratigraphie du secteur 20 n'a pu être établi et il s'agira de le préciser lors de la prochaine campagne, ce qui permettra d'assurer les liens existants entre les niveaux du secteur 20 et ceux plus à l'est sur le secteur 10, identifiés lors de la campagne 2019.

\section{La phase postérieure aux travaux du tribun T. Suedius Clemens (fin du troisième quart du Ier s. apr. J.-C. / 79 apr. J.-C.)}

Seules quelques années séparent l'époque des remaniements de la zone, liés à l'activité du Tribun Titus Suedius Clemens, de celle de l'éruption du Vésuve en 79 apr. J.-C. Durant cet intervalle, le niveau de circulation ne semble pas rehaussé, mais l'état de conservation de celui-ci ne permet pas d'identifier de nivellement ou de reprise dans l'emprise des fenêtres d'étude de cette campagne. Cependant, les journaux de fouilles $\mathrm{du}$ début du XX $\mathrm{X}^{\mathrm{e}}$ siècle indiquent notamment que la stèle SB10008 était partiellement recouverte par des déblais, tout comme les monuments funéraires de la zone 2 et certains espaces au pied des fortifications ${ }^{9}$. La présence de ces décharges sur des espaces publics indique que peu de temps après les opérations de restitutio du tribun et son départ ${ }^{10}$, la zone est encombrée par de vastes dépotoirs, évacués par les fouilleurs lors des premiers dégagements de cet espace périurbain.

\section{La zones 2 : secteur 20}

La zone 2 concerne les monuments funéraires installés à l'angle formé par l'axe viaire sortant de la ville en direction du nord et la voie "pomériale", longeant les fortifications à l'ouest en direction de la porte d'Herculanum (fig. 4). Elle est délimitée au nord par le mur méridional MR30003 de l'enclos ENC30001 (zone 3, secteur 30), dont les niveaux de fondation ont pu être appréhendés cette année. Lors de cette campagne, des investigations ont été entreprises au nord de la schola d'Arellia Tertulla (MSL20003) et autour du monument funéraire de Septumia (MSL20004). Les deux autres 
monuments funéraires de M. Veius Marcellus (MSL20001) et de C. Vestorius Priscus (MSL20002) n'ont pas été concernés par l'intervention programmée cette année.

Fig. 4. Plan de la zone 2 et des structures dégagées dans le secteur 20.

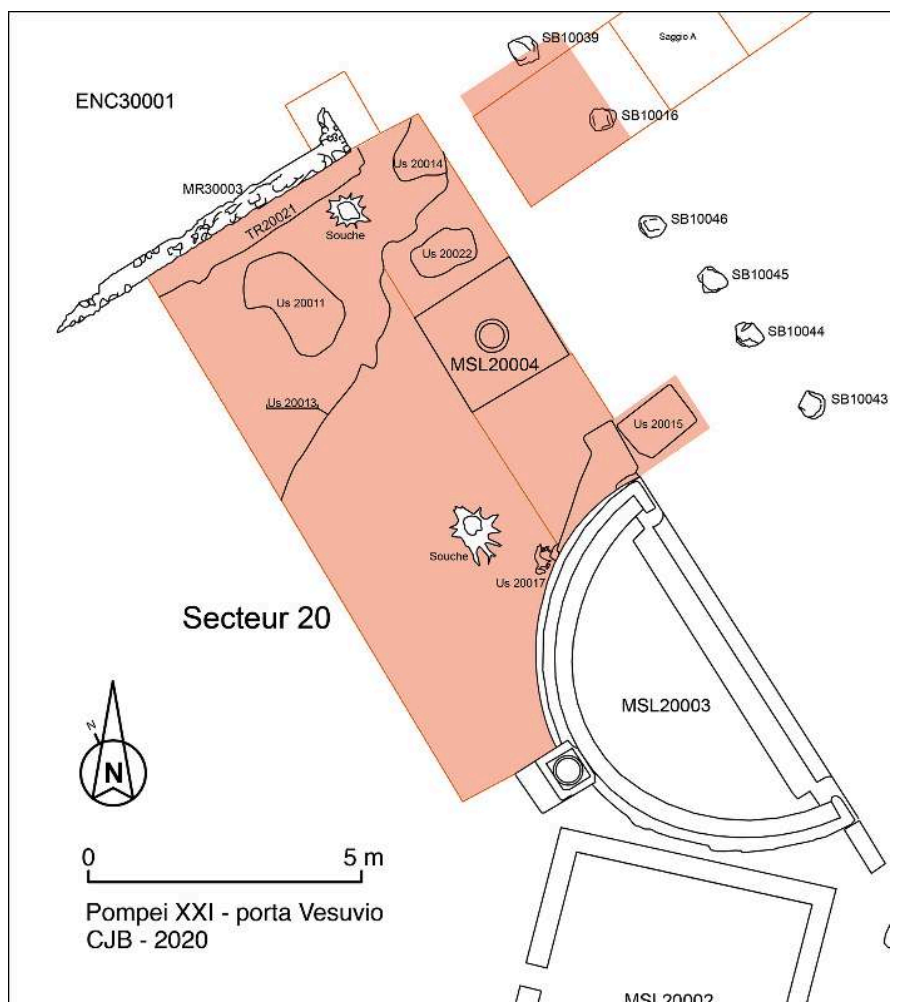

B. Lemaire, G. Chapelin, sur concession du MiC - PA Pompei. Tous droits réservés.

Lors de l'éruption du Vésuve en 79 apr. J.-C., ces monuments étaient partiellement recouverts par une vaste zone de dépotoirs, probablement issus du tremblement de terre de 62/63 apr.J.-C., comme mentionné précédemment. Cependant, en dehors d'anciennes photographies laissant deviner son existence en arrière-plan ${ }^{11}$, aucune trace n'en subsistait sur le terrain, jusqu'aux investigations de terrain menées en 2019 sur la zone $5^{12}$. Malheureusement, cette dernière n'a pu faire l'objet d'une fouille lors de cette campagne, mais d'autres découvertes réalisées sur le secteur 20 viennent apporter de premiers résultats.

\section{Les vestiges antérieurs à la fin du troisième quart du ler $\mathbf{s}$. apr. J.-C.}

Sur l'espace fouillé lors de cette campagne, les vestiges identifiés les plus anciens correspondent aux monuments funéraires MSL20003 et MSL20004 (fig. 5). Leurs niveaux de fondation respectifs n'ont pu être atteints, attestant leur antériorité par rapport aux niveaux appréhendés. Par conséquent, il s'agira d'atteindre ces niveaux de fondation lors des prochaines années, afin de préciser la chronologie de ces édifices, dont les études épigraphiques et stylistiques demeurent relativement imprécises ${ }^{13}$. Par exemple, V.L. Campbell propose une datation du monument d'Arellia Tertulla (MSL20003) entre 31 av. J.-C. et 14 apr. J.-C. ${ }^{14}$, alors que U. Soldovieri propose de le dater entre 41 apr. J.-C. et 60 apr. J.-C. ${ }^{15}$. De même, V. L. Campbell propose une datation de la tombe de Septumia (MSL20004) entre 31 av. J.-C. et 14 apr. J.-C. ${ }^{16}$, alors que U. Soldovieri 
propose de la dater entre 20 av. J.-C. et 10 apr. J.-C. ${ }^{17}$. Il s'agira donc de reprendre toutes les datations proposées par les chercheurs et de les confronter aux données stratigraphiques apportées par notre étude.

Fig. 5. Le secteur 20 en cours de fouille, vu de l'est.

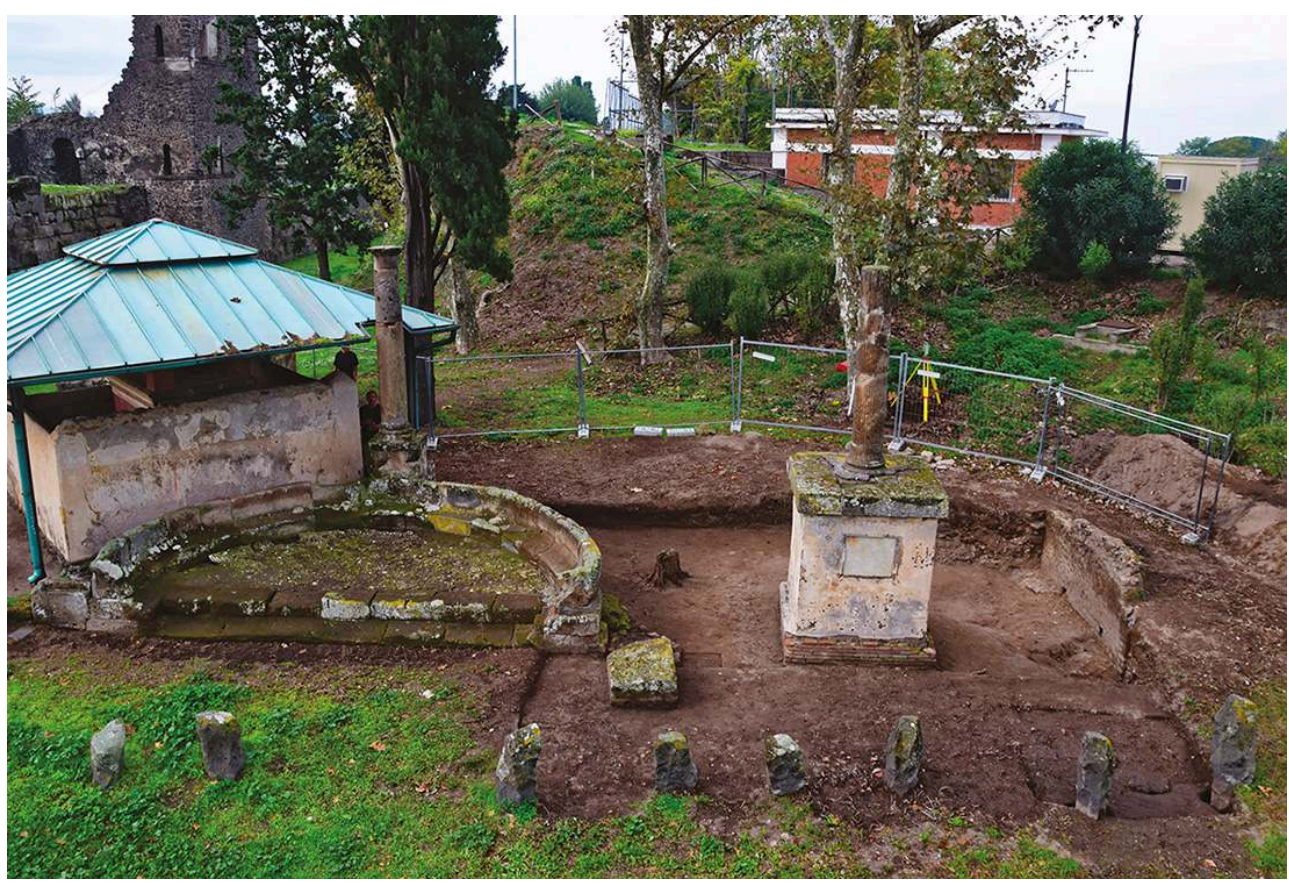

B. Lemaire, sur concession du MiC - PA Pompei. Tous droits réservés.

17 Sous les remblais contemporains et l'imposant dépotoir datant des derniers temps de Pompéi, plusieurs niveaux de fréquentation ont été appréhendés et sont postérieurs à l'installation des deux monuments funéraires MSL20003 et MSL20004. On retrouve, du plus ancien au plus récent :

- les sols SL20023 et SL20020 correspondent à un même niveau, mais dans deux sondages distincts, respectivement au nord et au sud du monument funéraire MSL20004. Non fouillés, ils présentent un limon brun moyen très compact, avec de petites inclusions de charbons et de quelques fragments de mortier, ainsi qu'un peu de fragments de céramique en surface ;

- les sols SL20022 et SL20018 correspondent à un même niveau, respectivement au nord et au sud du monument funéraire MSL20004. Ils présentent un limon sableux brun foncé avec de très nombreuses inclusions d'éclats de taille de tuf gris, ainsi que des fragments de céramique, de tuile, de faune, de métal et de rares enduits peints ;

- les sols SL20010 (= SL20019) et SL20012, correspondent à un même niveau, respectivement au nord et au sud du monument funéraire MSL20004. Ils présentent un limon brun moyen peu compact, ainsi que des fragments de céramique, de faune, de verre, de métal, d'enduit peint et des coquillages.

- Localisé au nord/nord-ouest du monument funéraire MSL20004 et recoupant les sols précédents, le creusement d'une vaste dépression (US 20013) a été identifié (fig. 6 et 7), avec une orientation sud-ouest/nord-est et plongeant vers le nord-ouest. Elle mesure jusqu'à 7,50 m de long (du sud-ouest au nord-est), 4,40 m de large et au maximum $60 \mathrm{~cm}$ de profondeur (au nord-est). C'est dans cette dépression que sera installé le mur MR30003, avant qu'elle soit comblée par une partie du vaste dépotoir US 20009 ; 
- enfin, le sol SL20025 correspond à une surface de fréquentation au-dessus des derniers niveaux de circulation SL20010 (= SL20019) et SL20012, ainsi que du creusement de la dépression US 20013. Ce sol pourrait dater de l'époque des remaniements menés par le tribun Titus Suedius Clemens, mais il n'a pas été possible d'établir un lien stratigraphique direct avec l'extrémité occidentale de la tranchée est-ouest réalisée l'an passé dans le secteur 10. Il s'agira de préciser ce point lors de la prochaine campagne.

Fig. 6. Le niveau de sol SL20012 autour du monument funéraire MSL20004 et la dépression US 20013 (à gauche), vus de l'ouest.

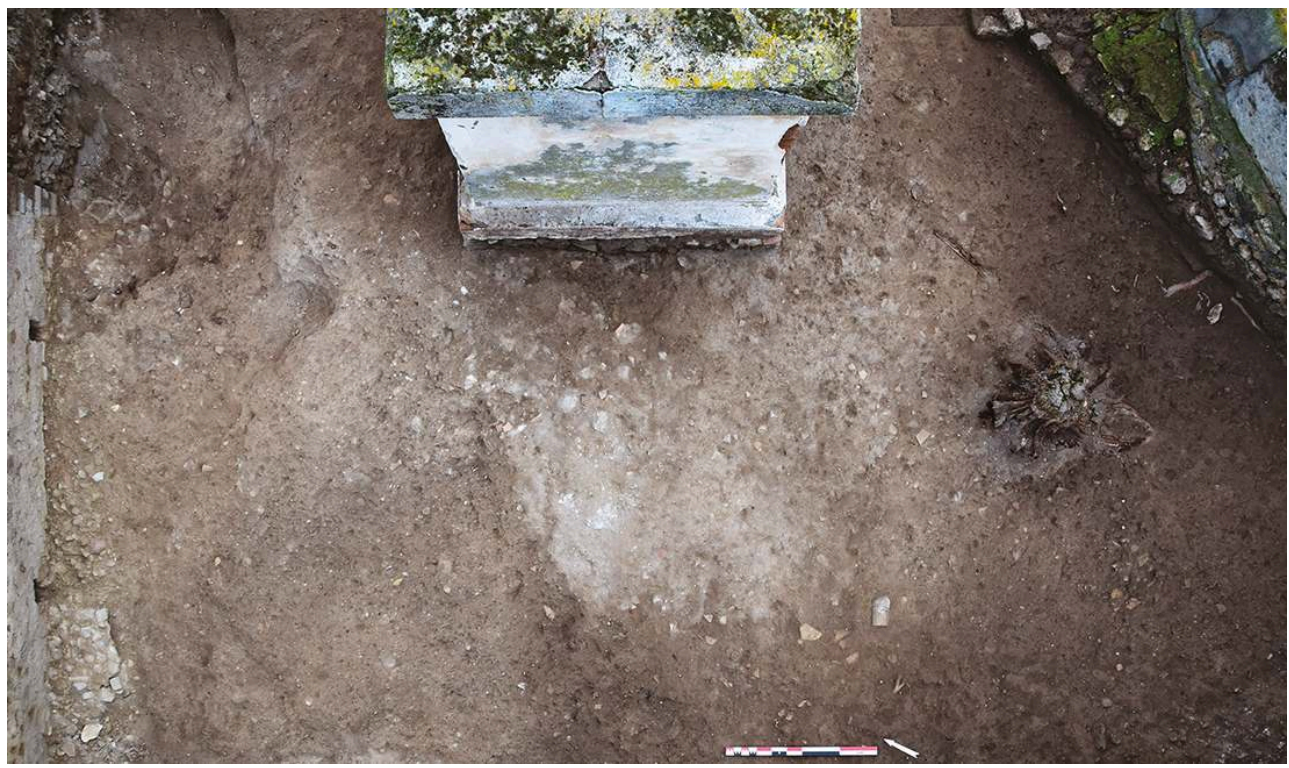

B. Lemaire, sur concession du MiC - PA Pompei. Tous droits réservés. 
Fig. 7. Courbes de niveau du secteur 20, avec le sol SL20012 et la dépression US 20013 au nordouest (bleu foncé), tirées du modèle photogrammétrique.

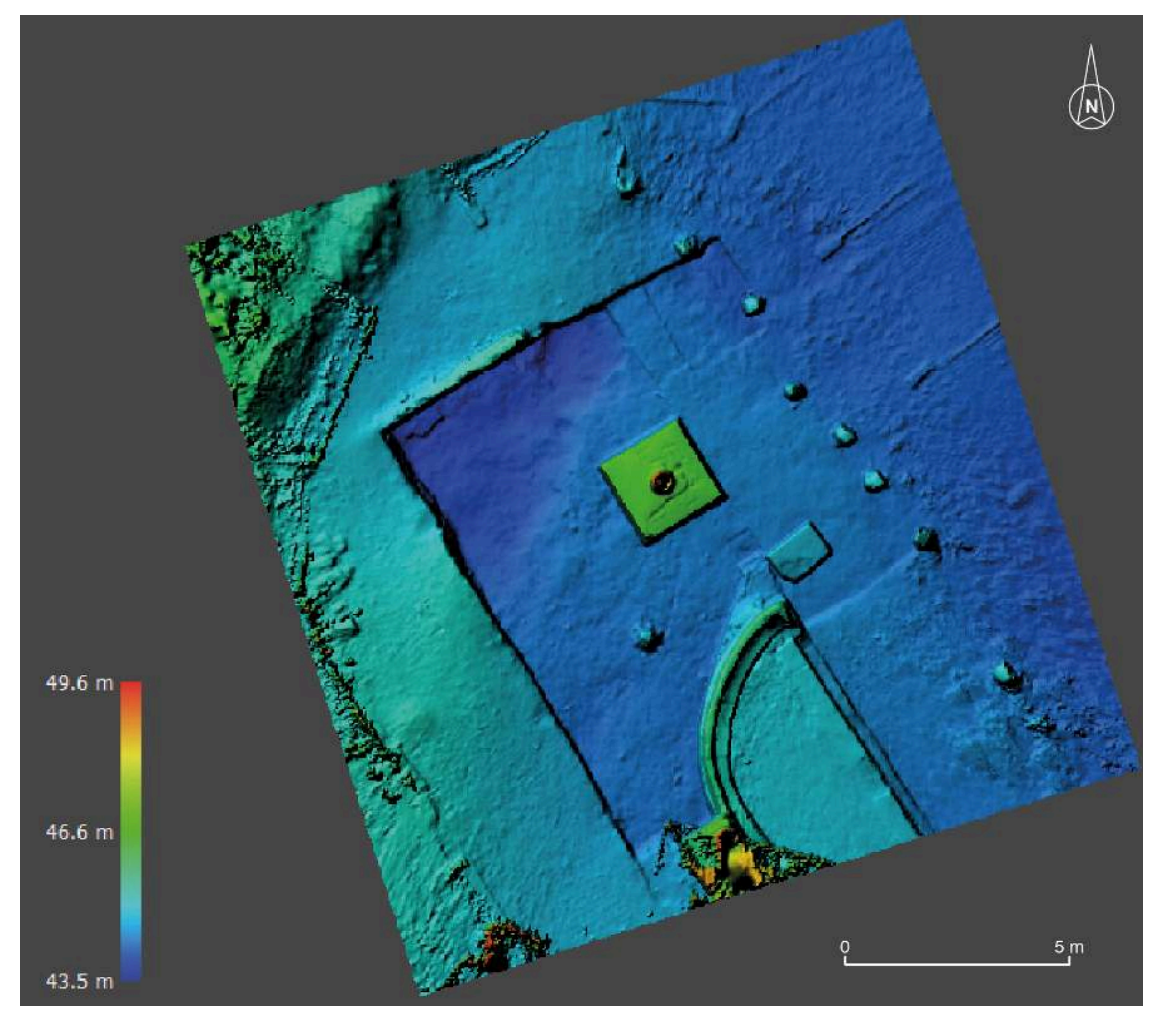

N. Leys, sur concession du MiC - PA Pompei. Tous droits réservés.

\section{Les travaux du tribun T. Suedius Clemens (fin du troisième quart du fer siècle} apr. J.-C.)

En plus des niveaux de fondation de la borne SB10039, marquant l'angle nord-est du secteur 20, d'autres installations pourraient être rapprochées des travaux engagés par le tribun Titus Suedius Clemens. En effet, le mur sud MR30003 de l'enclos ENC30001 et par conséquent le mur est MR30002 auquel il est chaîné semblent liés à ces remaniements, comme l'enclos ENC40001, de l'autre côté de la voie. Orienté est-ouest, le mur MR30003 a été suivi en plan sur 6,30 $\mathrm{m}$ lors de la campagne précédente et se poursuit plus à l'ouest. À l'est, l'angle formé avec le mur MR30002, orienté nord-sud, avait également pu être identifié. Cette année, le parement méridional du mur MR30003 a pu être dégagé sur 4,40 $\mathrm{m}$ de long et observé sur toute sa hauteur, depuis les fondations et jusqu'à l'arase et la réfection contemporaine (US 30008). Les photographies anciennes illustrent que les murs de cet enclos n'étaient pas très élevés, mais qu'une partie non négligeable de l'élévation a été endommagée, voire complètement détruite pour une partie du mur MR30002 ${ }^{18}$.

Le mur MR30003 est installé dans une tranchée TR20021 aux parois verticales de $58 \mathrm{~cm}$ de haut et large de 40 à $50 \mathrm{~cm}$ du côté sud. Celle-ci est creusée au fond de la dépression US 20013, dans la surface de fréquentation SL20025 et a été identifiée sur près de $4,20 \mathrm{~m}$, puis fouillée sur $2,90 \mathrm{~m}$ (fig. 8). Une semelle de fondation (US 20024) de mortier terreux brun clair, présentant de rares fragments de blocs de lave et de céramiques, a été installée au fond de la tranchée, sur laquelle une fondation maçonnée a été construite (US 30007). Celle-ci a été observée sur près de $60 \mathrm{~cm}$ de haut et 
présente la même technique que le reste de l'élévation, mais pas de joints lissés en surface. La tranchée TR20021 est ensuite comblée par le niveau US 20016, constitué d'un limon sableux brun clair, présentant du matériel (céramique, verre, faune, fer, enduits peints, TCA). Le sommet de la couche est compact et présente des résidus de mortier en surface, en relation avec la construction de l'élévation du mur MR30003 et la réalisation de joints lissés.

Fig. 8. Le mur MR30003 et sa tranchée de fondation TR20021, vus du sud.

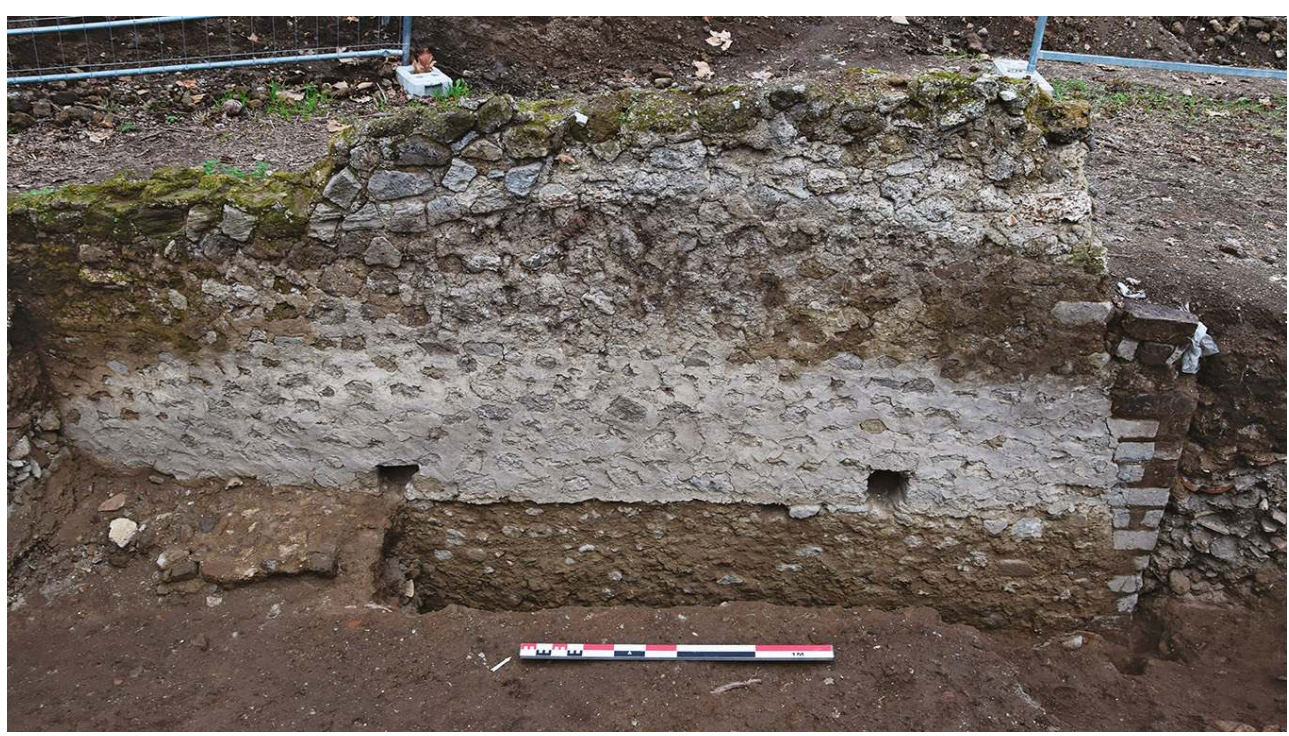

B. Lemaire, sur concession du MiC - PA Pompei. Tous droits réservés.

moellons irréguliers de lave, de quelques calcaires du Sarno et de rares fragments de sol et de mortier, liés dans un mortier de terre brun clair. À l'extrémité orientale, le chaînage en moellons rectangulaires a été observé depuis sa fondation. L'élévation du mur MR30003 mesure jusqu'à $40 \mathrm{~cm}$ d'épaisseur, a été observée sur plus de 1,30 m de hauteur et présente deux barbacanes dans sa partie inférieure, à la liaison avec la fondation et le comblement de la tranchée. Servant très probablement à évacuer l'excédent d'eau de l'enclos ENC30001, ces barbacanes sont espacées de 2,45 m et mesurent $14 \mathrm{~cm}$ de large pour $15 \mathrm{~cm}$ de haut du côté ouest et $15 \mathrm{~cm}$ de large pour $17 \mathrm{~cm}$ de haut du côté est. L'ensemble du parement de l'élévation (US 30003) présente des joints lissés et bien marqués (US 30006), réalisés en mortier gris clair. Ceux-ci ne sont pas présents sur la fondation (US 30007), mais reviennent légèrement sur la partie supérieure du comblement US 20016 de la tranchée de fondation TR20021.

\section{La phase postérieure aux travaux du tribun T. Suedius Clemens (fin du troisième quart du ler siècle apr. J.-C. / 79 apr. J.-C.)}

21 antique ont été appréhendés, mais apparaissent relativement mal conservés en surface en raison des dégradations effectuées à l'époque contemporaine. En effet, au moment de l'éruption du Vésuve, l'imposant niveau de décharge qui recouvrait les monuments funéraires, probablement issu du tremblement de terre de 62/63 apr.J.-C., a été largement évacué lors des premiers dégagements du début $d u X X^{e}$ siècle $^{19}$. Il ne reste 
qu'une partie réduite et aplanie en surface de ce vaste dépotoir (US 20009), que nous avons pu identifier et fouiller autour du monument funéraire MSL20004 et au nord de MSL20003 (fig. 9-10). Bien que ce niveau soit majoritairement conservé sur une épaisseur ne dépassant pas $10 \mathrm{~cm}$, l'existence d'une vaste dépression (US 20013) au nord-ouest du secteur a cependant permis de l'appréhender sur une hauteur allant jusqu'à $60 / 75 \mathrm{~cm}$. La couche est présente sur l'ensemble du secteur et vient contre le mur MR30003 de l'enclos ENC30001 au nord et contre le bâti des monuments funéraires MSL20003 et MSL20004, impliquant leur antériorité.

Fig. 9. Le niveau de dépotoir US 20009 autour du monument funéraire MSL20004, vu du nord.

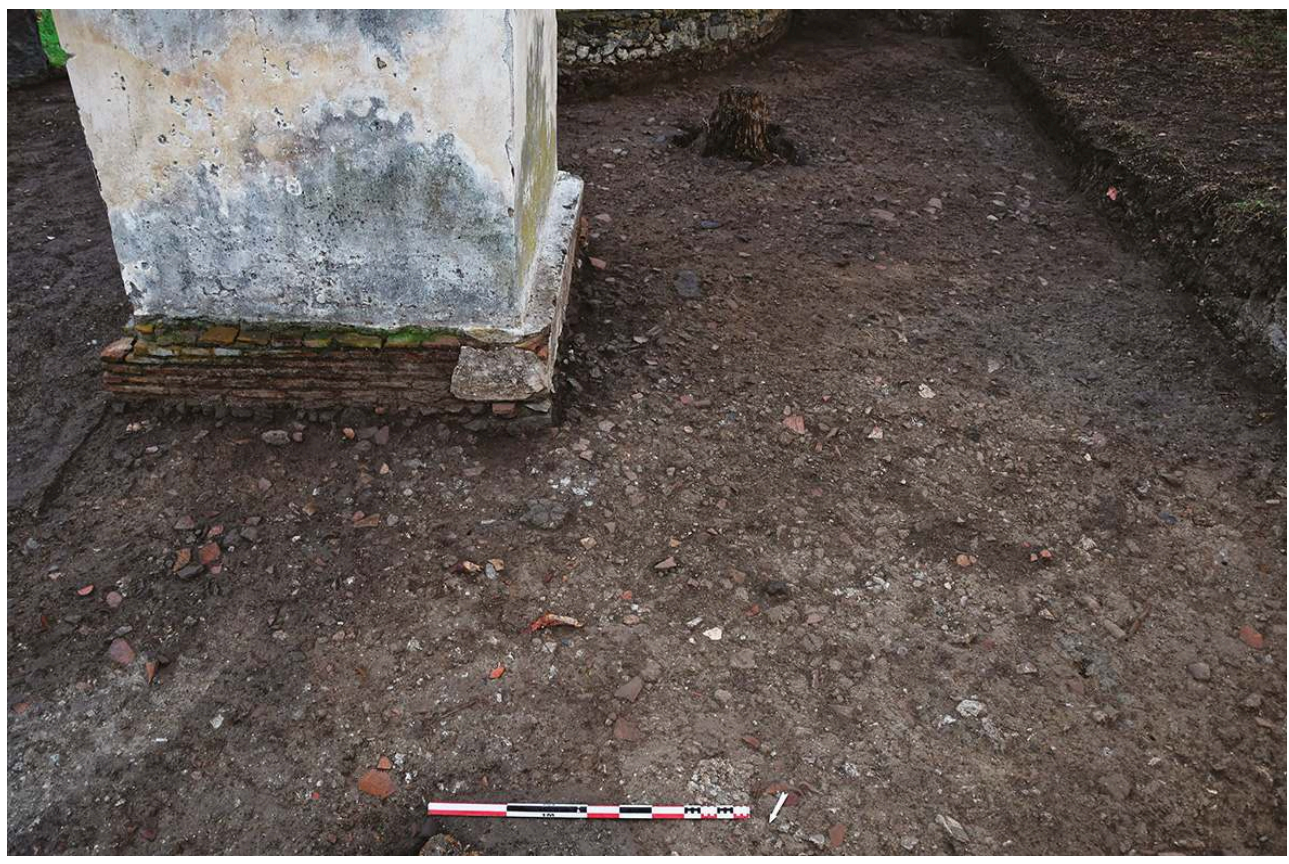

B. Lemaire, sur concession du MiC - PA Pompei. Tous droits réservés. 
Fig. 10. Détail du dépotoir US 20009 en cours de fouille, à l'ouest du monument funéraire MSL20004.

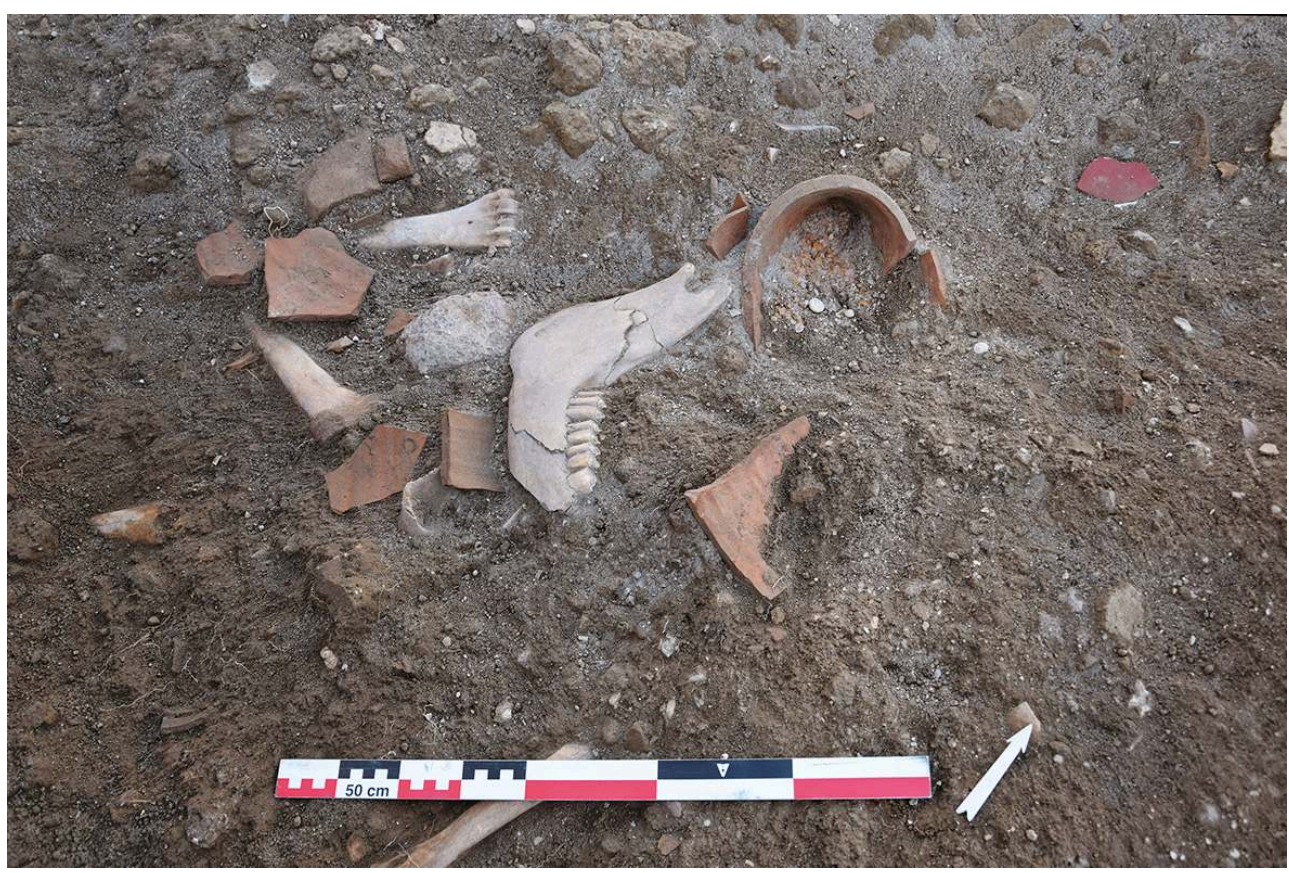

B. Lemaire, sur concession du MiC - PA Pompei. Tous droits réservés.

Ce niveau US 20009 est composé d'une matrice limono-sableuse brun moyen à gris, peu compacte et présente des éléments issus de la destruction de bâtiments (fragments de mortier informes, de sols en béton de tuileau, de mortier de toiture, de tuiles, rares petits et moyens blocs et très rares éléments maçonnés). Une très grande quantité de matériel antique a été identifiée et prélevée : des fragments de céramique (amphore, parois fines, culinaire, lampes, etc.), de faune, d'enduit peint (uni ou multicolore), de verre, de métal (fer et bronze principalement), six monnaies, ainsi que des petits objets particuliers (dont une fibule en bronze, 1 pendentif en cristal de roche, 1 moitié de bijou en gemme violet, etc.). Un fond d'amphore contenant très probablement les restes de préparation de garum (écailles et arêtes de poissons) a également été découvert.

À l'intérieur de ce remblai de type dépotoir et dans l'emprise de la dépression (US 20013) au nord du secteur, là où l'état de conservation est le plus important, deux couches techniques ont pu être individualisées (US 20011 et US 20014). Le niveau US 20011 est un limon cendreux gris avec de très nombreux restes d'ossements de faune brulés et présentant un pendage vers le nord. Il est localisé à l'ouest dans la dépression (US 20013) et mesure 2,00 $\mathrm{m}$ du nord au sud et $1,10 \mathrm{~m}$ d'est en ouest. Le niveau US 20014 (fig. 11) est constitué de charbons, présente un pendage vers l'ouest et est localisé à l'extrémité orientale de la dépression (US 20013) et au nord du monument funéraire MSL20004. Il mesure 1,05 $\mathrm{m}$ du nord au sud et 0,95 $\mathrm{m}$ d'est en ouest. 
Fig. 11. La couche technique US 20014 faisant partie du dépotoir US 20009, au nord du monument funéraire MSL20004, vue de l'ouest.

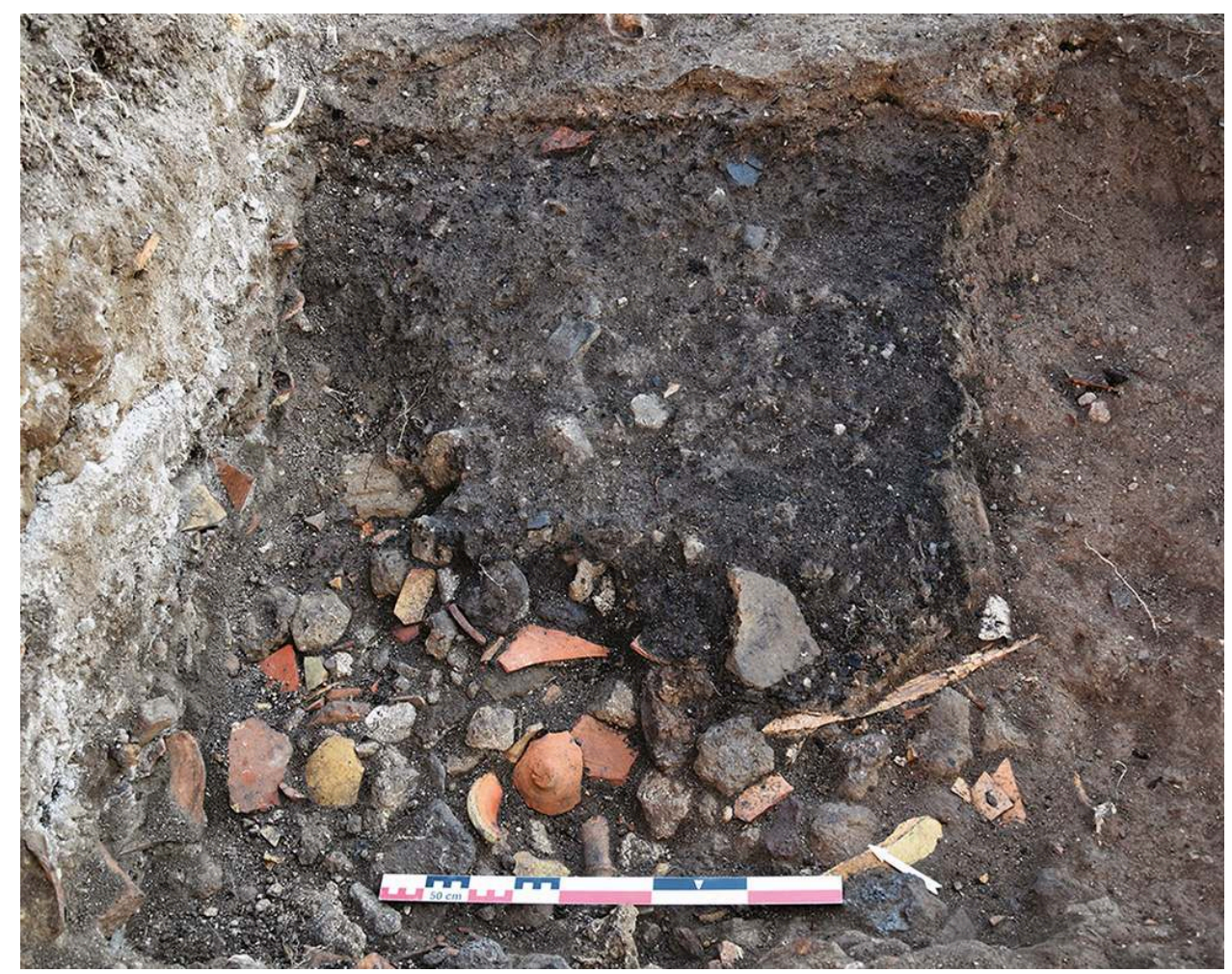

B. Lemaire, sur concession du MiC - PA Pompei. Tous droits réservés. apr. J.-C., l'absence de blocs issus de la destruction de bâtiments - alors que de très nombreux éléments associés à ce type de construction ont été découverts (fragments de mortier, de tuile, etc.) - implique qu'un tri a été effectué en amont et que la très grande majorité des blocs a été récupérée afin de reconstruire les édifices de Pompéi. Seuls les éléments difficilement ou non réutilisables semblent avoir été déposés dans cette décharge à la sortie de la ville. notamment à évaluer le volume de la portion étudiée du dépotoir (US 20009, US 20011 et US 20014) et permettra ainsi de réaliser des statistiques précises suite à l'étude du matériel.

Enfin, directement sur le sol SL20025, sur lequel repose le dépotoir US 20009, les restes d'un canidé en connexion (US 20017) ont été identifiés (fig. 12), au sud-ouest du monument MSL20004 et contre le parement de la schola MSL20003. Orienté nord-sud, celui-ci est en position latérale sur le flanc droit, la tête vers le sud. La mâchoire est fermée et tournée vers le monument MSL20003, tout comme les pattes. Des perturbations racinaires ont été identifiées au niveau de l'abdomen et aucun creusement n'a été identifié. 
Fig. 12. Les ossements du chien US 20017, vus du nord.

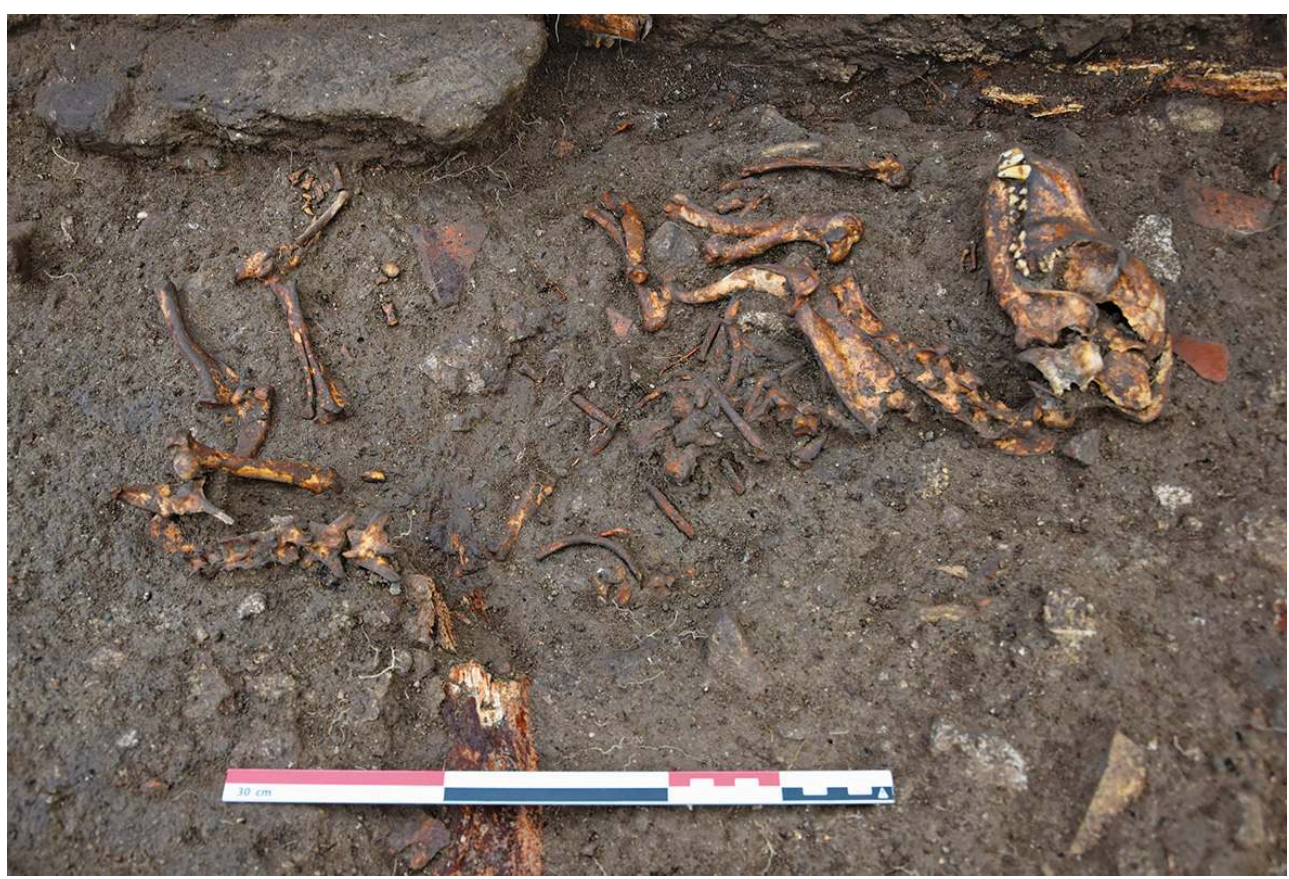

B. Lemaire, sur concession du MiC - PA Pompei. Tous droits réservés.

\section{Les perturbations contemporaines}

Dans le secteur 20, une série de niveaux contemporains et d'interventions postérieures aux dégagements du début du $\mathrm{XX}^{\mathrm{e}}$ siècle ont été reconnus :

- un niveau de sol contemporain (SL20008) a été identifié sur l'ensemble des couches antiques et notamment sur les restes du dépotoir antique (US 20009). Ce dernier a été largement évacué lors des premiers dégagements du début du XXe siècle et n'a donc pas fait l'objet d'études approfondies, laissant la zone dans une situation qui ne s'apparentait plus à celle qui devait exister en 79 apr. J.-C. Ce sol SL20008 (fig. 13), témoin de la fréquentation de l'espace après les premiers dégagements de la zone, est constitué d'un limon brun moyen, peu compact, présentant des traces de colluvionnements, ainsi que du matériel antique et contemporain. C'est sur ce sol SL20008 qu'un bloc (US 20015), appartenant au monument funéraire MSL20003 et localisé à son extrémité nord-est, est tombé à l'époque contemporaine. En effet, celui-ci apparait en place sur les anciennes photographies. Sa restauration est envisagée en collaboration avec les services de la Surintendance ;

- un imposant remblai de terre contemporaine (US 20005) recouvrait l'ensemble du secteur, composé d'un limon brun foncé meuble, de quelques blocs et présentant du matériel antique et contemporain, dont deux pipes en terre cuite. Deux couches techniques ont été individualisées dans ce niveau, présentant également du matériel antique et contemporain. La couche US 20007, localisée au nord-ouest du monument funéraire MSL20004 et recouvrant le sol SL20008, est composée d'un limon brun moyen à gris et très compact en surface (jusqu'à 3,05 $\mathrm{m}$ du nord au sud et 3,80 $\mathrm{m}$ d'est en ouest). Des lapilli gris et de rares lapilli blancs ont été identifiés, ainsi qu'un fragment de colonne en tuf gris. La couche US 20006, située au nord-ouest du secteur 20, se compose d'une grande quantité de lapilli blancs et de quelques petits blocs, pris dans une matrice de limon brun foncé $(1,60 \mathrm{~m}$ du nord au sud, 1,30 m d'est en ouest et jusqu'à $8 \mathrm{~cm}$ d'épaisseur); 
- enfin, il faut noter que la zone a été bombardée par les Alliés en $1943^{20}$ et qu'au moins la partie occidentale du monument funéraire de M.Veius Marcellus (MSL20001) a été partiellement détruite, puis reconstruite.

Fig. 13. Le niveau de sol SL20008, vu du nord.

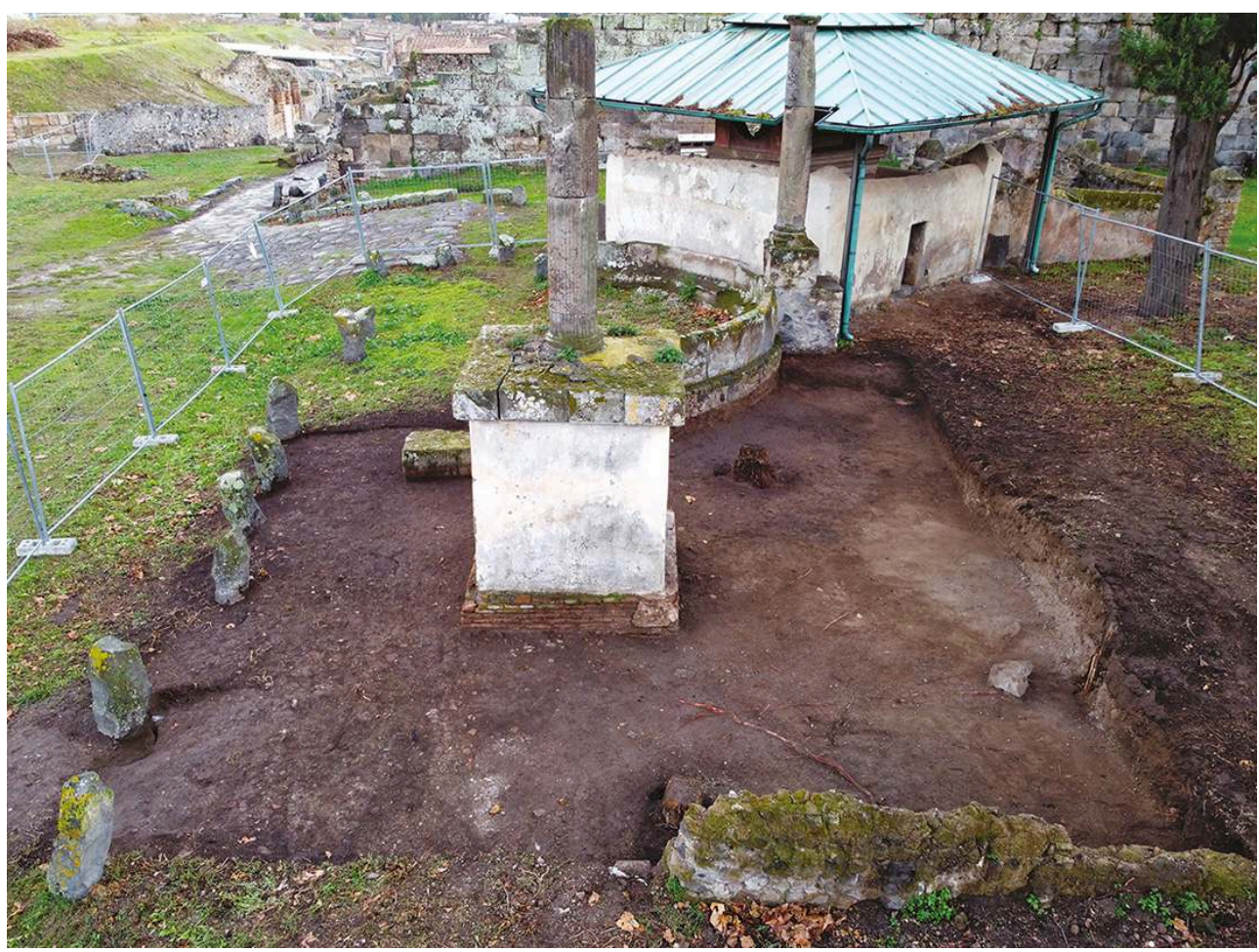

B. Lemaire, sur concession du MiC - PA Pompei. Tous droits réservés.

\section{La zone 4 : secteurs 40 et 41}

La zone 4 concerne l'espace bordant la voie partant vers le nord (zone 1) sur son côté oriental et est limitée à l'ouest par le mur MR40001. Le secteur 41 a été ouvert cette année (fig. 14), dans le grand enclos ENC40001 identifié en bordure de la voie et vient compléter les données apportées par les investigations menées l'année dernière, notamment par les sondages effectués au pied du mur MR40001 dans le secteur 10. 
Fig. 14. Plan de la zone 4 et des structures dégagées dans le secteur 41 .

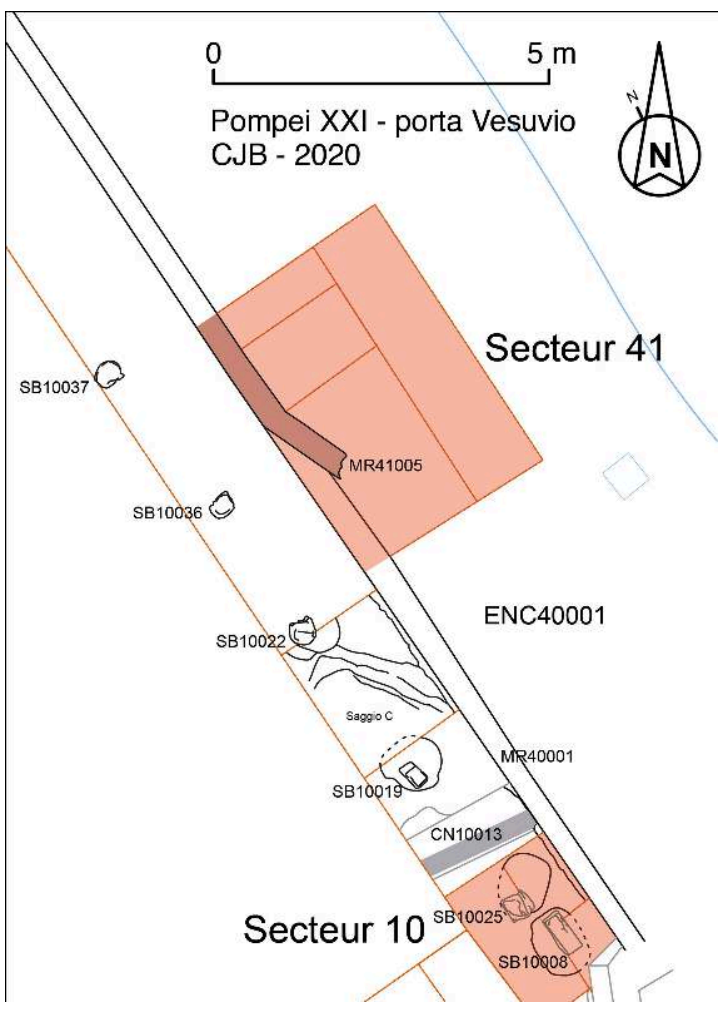

B. Lemaire, G. Chapelin, sur concession du MiC - PA Pompei. Tous droits réservés.

Le secteur 41 mesure $4,56 \mathrm{~m}$ du nord au sud et jusqu'à $2,90 \mathrm{~m}$ d'est en ouest, pour une surface de plus de $13 \mathrm{~m}^{2}$ (fig. 15). Il est bordé à l'ouest par le mur MR40001 et un palier de sécurité de $1,20 \mathrm{~m}$ a dû être réalisé du côté oriental afin de pouvoir atteindre les niveaux antiques. 
Fig. 15. Orthophotogrammétrie du secteur 41 .

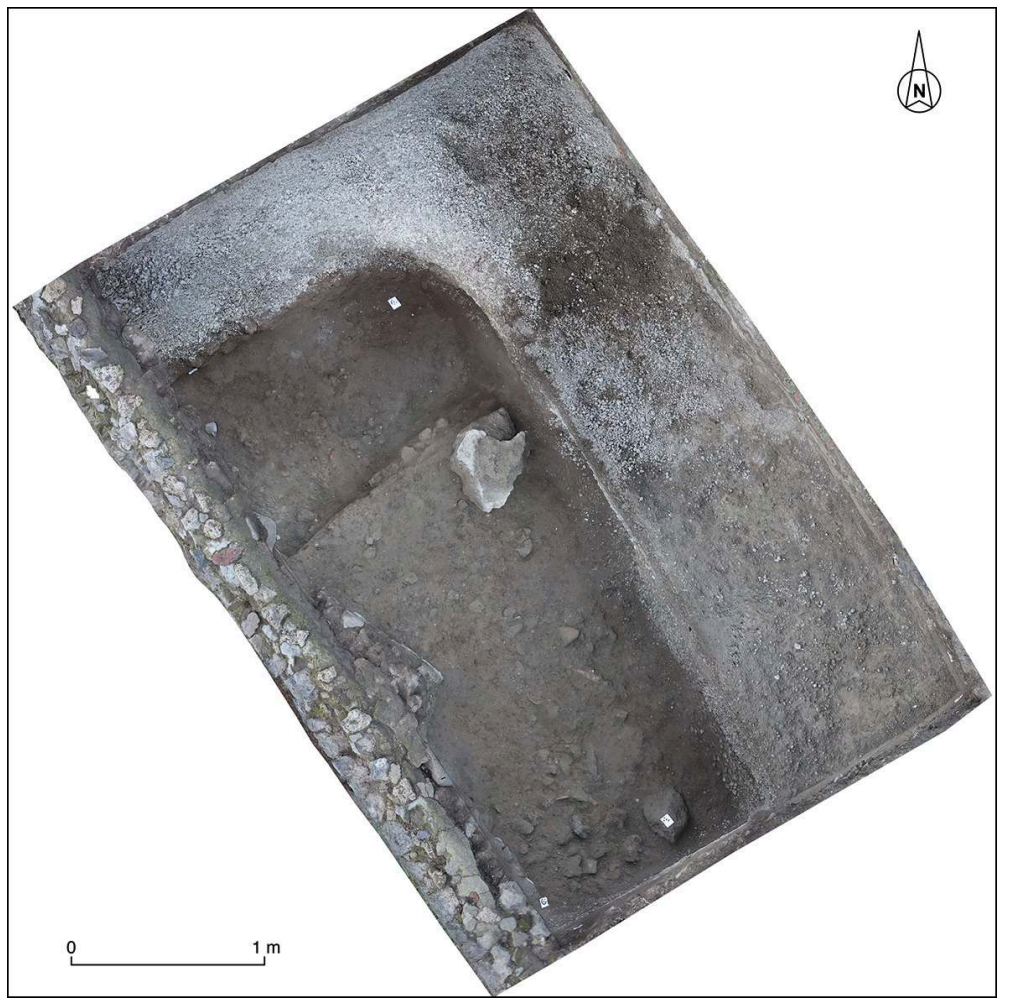

B. Lemaire, N. Leys, sur concession du MiC - PA Pompei. Tous droits réservés.

Les vestiges antérieurs à la fin du troisième quart du ler $\mathbf{s}$. apr. J.-C.

Les vestiges les plus anciens identifiés dans le secteur sont représentés par le mur MR41005. Il est orienté nord-sud sur la partie nord et bifurque vers le sud-est à partir du centre du sondage. Ce mur servira de fondation au mur MR40001, dans la partie nord, alors que sa partie sud sera détruite (fig. 16). Le mur MR41005 mesure 34 à $38 \mathrm{~cm}$ de large et a été observé sur 2,20 m de long et 1,90 m de haut. Il est réalisé en petits blocs de lave, avec quelques calcaires du Sarno, de rares petits blocs de cruma, quelques fragments de tuiles et un peson en céramique, liés avec un mortier de terre présentant quelques gros nodules de chaux. Il est donc possible d'avancer qu'à partir de la moitié du sondage, un premier mur bifurquait vers le sud-est, alors que les précédentes études menées d'après les images d'archives et les investigations de la campagne 2019, n'en laissaient aucunement supposer l'existence. 
Fig. 16. Les parements orientaux des murs MR40001 et MR41005, vus de l'est.

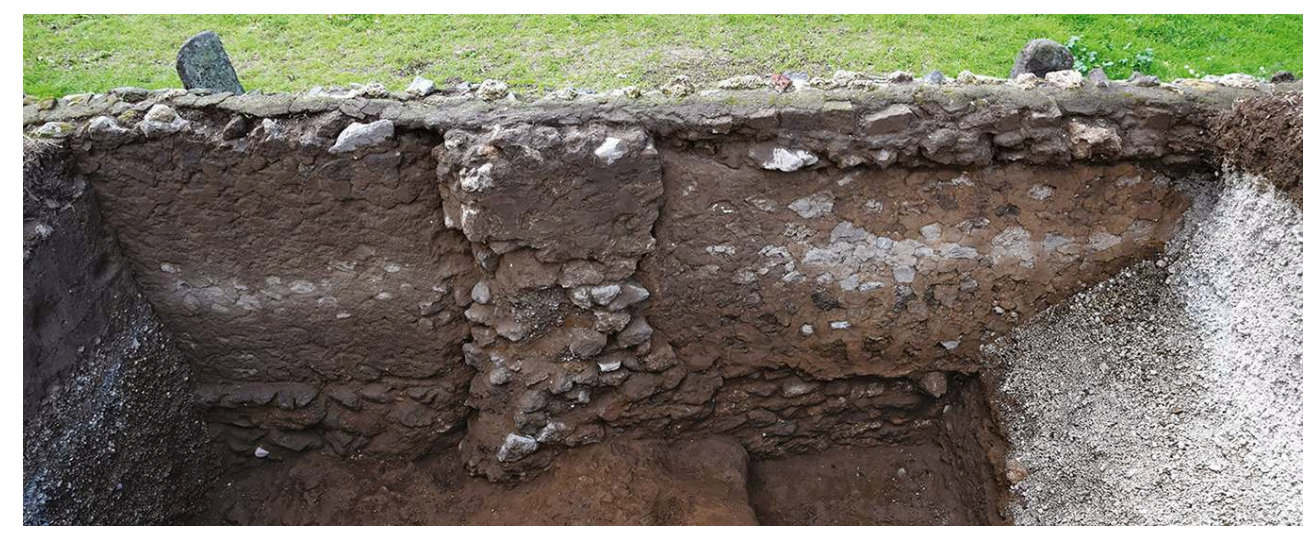

B. Lemaire, sur concession du MiC - PA Pompei. Tous droits réservés.

31 Les niveaux de fondation du mur MR41005 n'ont pu être atteints pour des raisons de sécurité, mais une couche (US 41007) a pu être identifiée, venant contre son parement oriental. Celle-ci est antérieure aux travaux du tribun Titus Suedius Clemens et n'a été que très partiellement fouillée, dans l'emprise du sondage réduit effectué au nord-ouest du secteur 41. Elle présente un pendage vers l'est et se compose d'un limon brun/gris peu compact, avec des inclusions de charbons et des fragments de céramique, de métal, de faune, de verre et de mortier.

\section{Les travaux du tribun T. Suedius Clemens (fin du troisième quart du $\mathrm{I}^{\mathrm{er}} \mathbf{s}$.} apr. J.-C.)

32 C'est dans le niveau US 41007, précédemment décrit, qu'intervient l'installation du mur MR40001 datant des interventions menées par le tribun Titus Suedius Clemens. Le tracé du mur antérieur MR41005 est modifié. Sa partie sud, bifurquant vers le sud-est, est détruite (arrachement: US 41011) et sa partie nord conservée sert de fondation à un nouveau mur (MR40001), qui est prolongé vers le sud, jusqu'à un retour à angle droit marquant la limite sud de l'enclos ENC4000121. Les joints du parement oriental du mur MR41005 sont refaits à ce moment-là - sur la hauteur visible de celui-ci - afin d'unifier la construction avec le mur MR40001.

33 Les niveaux de fondation du mur MR40001 ont pu être identifiés et mis en relation avec ceux appréhendés lors de la campagne 2019, du côté occidental du mur. Ainsi, on retrouve une tranchée de fondation TR41009 (=TR40004) aux parois irrégulières et servant à l'installation de la fondation coulée US 41006 (= Us 40003). Cette dernière est réalisée en petits blocs de lave et d'ignimbrite campana (faciès gris), noyés dans un mortier de terre et de chaux à gros nodules. Cette fondation est débordante, jusqu'à $8 \mathrm{~cm}$ par rapport au parement oriental du mur MR40001 et a été observée sur 1,22 $\mathrm{m}$ de long et au maximum $52 \mathrm{~cm}$ de haut, mais se poursuit plus profondément. Sur cette fondation coulée, on retrouve un ressaut de fondation US 41008 (= Us 40002) servant d'appui à l'élévation du mur MR40001, dépassant jusqu'à $8 \mathrm{~cm}$ par rapport au parement oriental du mur.

34 Le mur MR40001, orienté nord-sud ferme l'enclos ENS40001 sur son côté ouest et a été observé sur $31,20 \mathrm{~m}$, mais ses extrémités nord et sud ne sont pas déterminées. Ce mur est restauré dans sa partie supérieure. Il mesure entre 34 et $38 \mathrm{~cm}$ de large, est réalisé en opus incertum et on note une tendance à la réalisation d'assises horizontales. Il est 
constitué de blocs de taille moyenne, dégrossis, de calcaire du Sarno, de lave, de cruma et d'ignimbrite campana (faciès gris). Ces éléments sont liés avec un mortier à base de terre et d'un peu de chaux, de couleur marron clair.

\section{La phase postérieure aux travaux du tribun T. Suedius Clemens (fin du troisième quart du Jer siècle apr. J.-C. / 79 apr. J.-C.)}

Venant contre le parement rejointoyé du mur MR41005 et le mur MR40001, un niveau US 41004 a pu être identifié (fig. 17). Celui-ci témoigne des dernières années d'occupation avant l'éruption du Vésuve en 79 apr.J.-C., car il est directement recouvert par les niveaux éruptifs US 41003 . Le remblai US 41004 a été partiellement fouillé et présente un fort pendage vers l'est et le sud-est. Il s'agit d'un limon brun moyen pulvérulent, présentant de rares petits blocs de lave, quelques fragments de mortier de terre, de tuiles, de céramique et de faune. Un bloc en calcaire du Sarno a été découvert en surface de cette couche ( $48 \times 48 \times 24 \mathrm{~cm})$.

Fig. 17. La couche US 41004 observée sous les niveaux éruptifs de 79 apr. J.-C., vue de l'est.

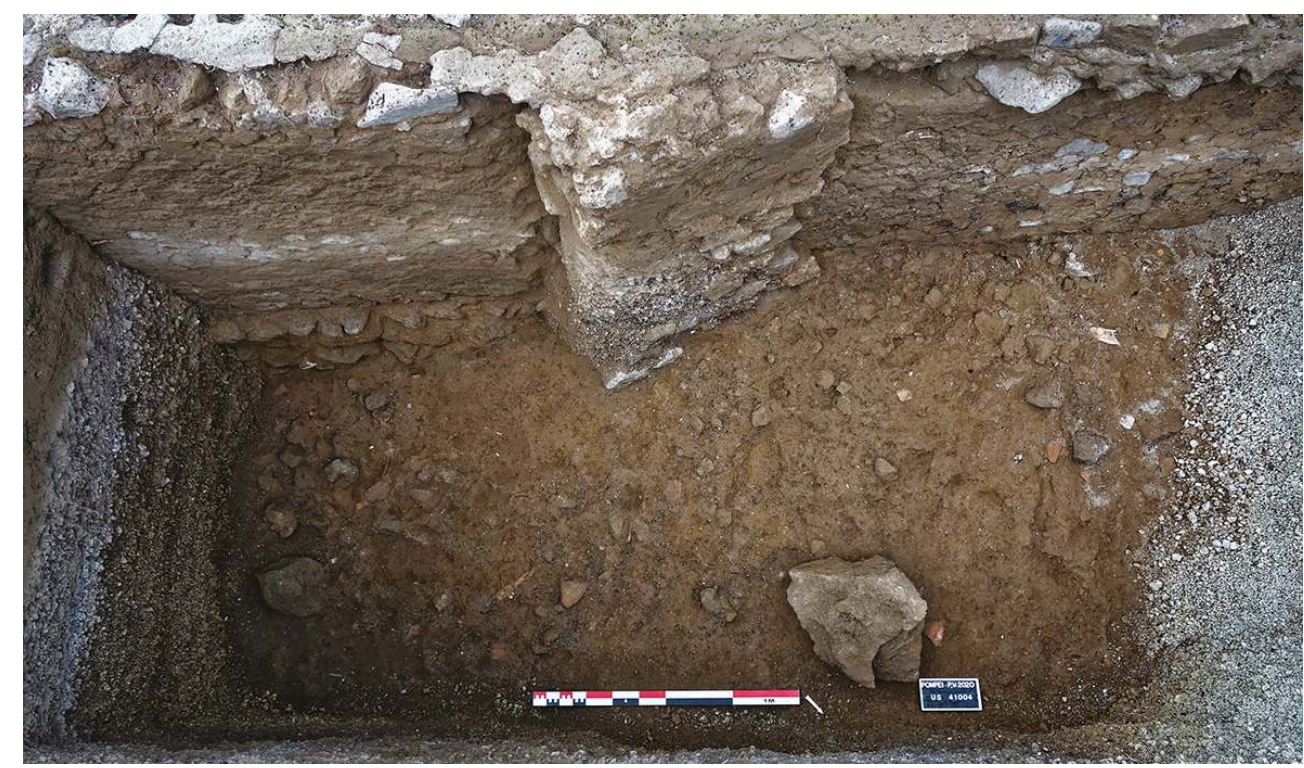

B. Lemaire, sur concession du MiC - PA Pompei. Tous droits réservés.

On remarque que la partie méridionale de la couche US 41004 a dû être partiellement décaissée (US 41010) durant l'Antiquité, car la partie supérieure de la fondation coulée (US 41006) du mur MR40001 était visible avant l'éruption du Vésuve.

\section{Les niveaux de l'éruption de 79 apr. J.-C.}

Bien que majoritairement dégagés au début du $\mathrm{XX}^{\mathrm{e}}$ siècle, les restes du niveau de l'éruption (US 41003) ont été identifiés sur l'ensemble du sondage. En raison de l'avancée des dégagements contemporains, cette couche possède un pendage vers le sud de plus de $90 \mathrm{~cm}$. Celui-ci correspond en réalité à l'avancée des dégagements réalisés au début $\mathrm{du} \mathrm{XX}^{\mathrm{e}}$ siècle, comme l'illustre une photographie aérienne datant de $1910^{22}$. La couche US 41003 est constituée de lapilli blancs et de quelques petits éléments volcaniques plus foncés, denses et lourds. La partie recouverte par la couche US 41002 ( $3 / 4$ sud) apparait plus grise car il y a eu un phénomène d'infiltration. Cette 
couche US 41003 (fig. 18) est conservée sur 1,75 m de haut au nord, mais était bien plus haute à l'origine.

Fig. 18. Vue de la paroi sud du secteur 41 , d'après le modèle photogrammétrique.

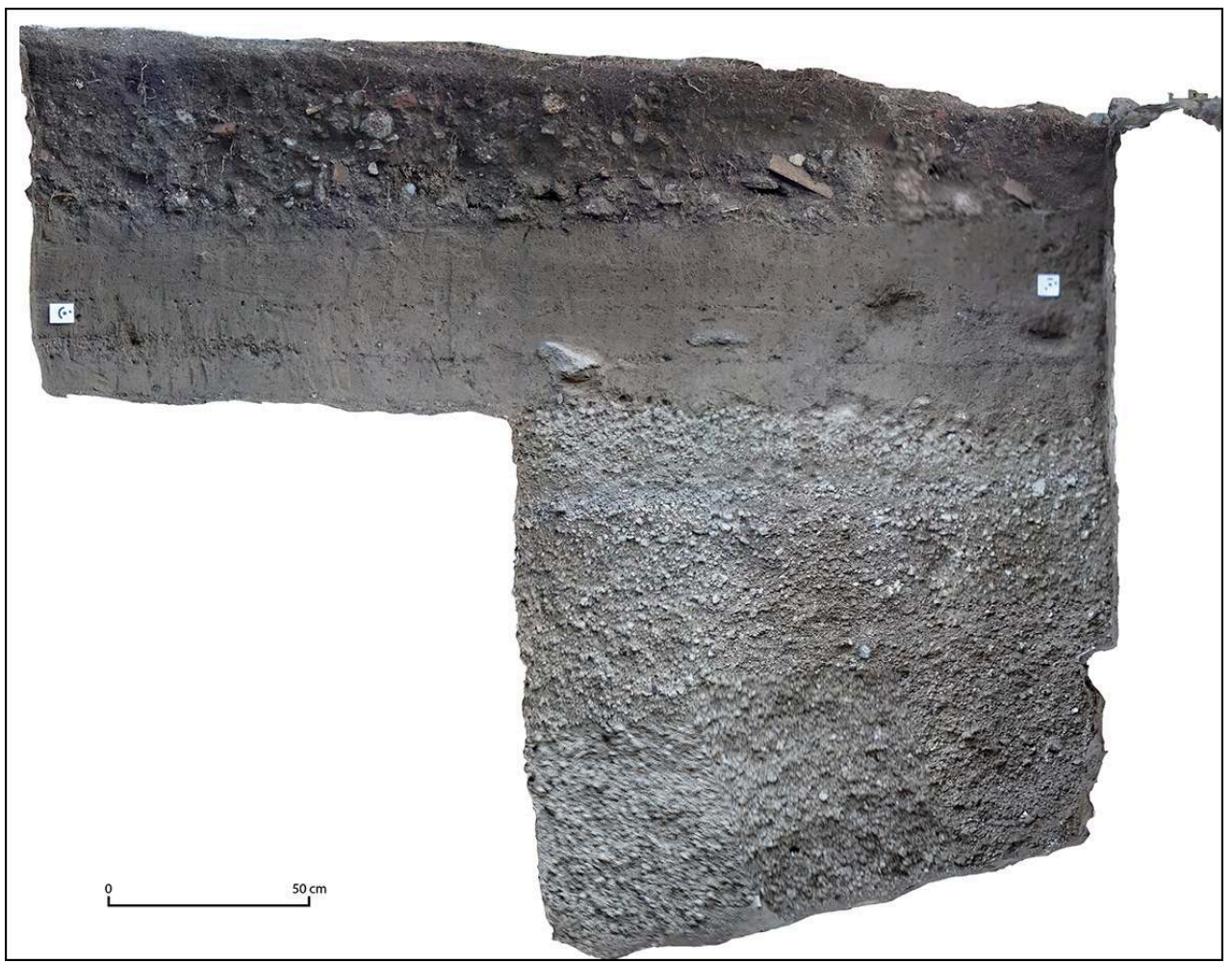

Trois niveaux se succèdent de haut en bas : deux niveaux contemporains US 41001 et US 41002, ainsi qu'un niveau de lapilli (US 41003) datant de l'éruption de 79 apr. J.-C.

B. Lemaire, N. Leys, sur concession du MiC - PA Pompei. Tous droits réservés

\section{Les perturbations contemporaines}

Recouvrant les niveaux éruptifs de 79 apr. J.-C., deux couches contemporaines ont été identifiées (fig. 18). On retrouve tout d'abord un niveau de limon brun cendreux compact (US 41002), qui mesure entre 4 et $60 \mathrm{~cm}$ d'épaisseur, du nord au sud. Il repose sur une partie de la couche de lapilli blancs (US 41003), dont il corrige le pendage. Enfin, un imposant remblai de terre contemporaine (US 41001) recouvrait l'ensemble du secteur, composé d'un limon brun foncé peu compact, de quelques blocs divers, dont du mortier et du béton. Il mesure entre 40 et $50 \mathrm{~cm}$ d'épaisseur et présente du matériel contemporain.

\section{Conclusion}

La réinterprétation des documents concernant les anciens dégagements, à la lumière des investigations de terrain de cette seconde campagne de fouille, autorisent à mieux cerner les aménagements situés en avant de la porte du Vésuve et notamment autour de la voie partant vers le nord, encadrée par des bornes, deux enclos et quatre monuments funéraires. 

l'inscription du tribun Titus Suedius Clemens, a permis la réalisation d'un modèle photogrammétrique, autorisant à en tirer des modèles précis et à l'intégrer à terme dans un modèle interactif.

41 dépotoir évacué lors des dégagements du début du XXe siècle et la fouille d'une partie de celui-ci devait permettre de mieux cerner l'origine des déblais et leur possible lien avec le tremblement de terre de 62/63 apr.J.-C. Bien qu'il n'ait pas été possible d'ouvrir le sondage prévu cette année, la fouille réalisée dans la zone 2 apporte déjà de nouvelles données. En effet, la fouille des restes de l'imposant dépotoir antique, laissés par les fouilleurs du début $\mathrm{du} \mathrm{XX}^{\mathrm{e}}$ siècle dans le secteur 20 et datant des dernières années de Pompéi, apporte d'importants éléments de réflexion sur la nature de ces remblais, sur le tri qui a pu être opéré en amont, sur l'étendue chronologique des éléments le constituant, etc. Cependant, une campagne dédiée à l'étude du matériel devra être réalisée au cours de l'année 2021, afin de poursuivre l'étude de ce dépotoir, qui n'avait jusque-là que peu retenu l'attention des chercheurs.

42 Le sondage réalisé dans la zone 4 a permis de mieux cerner les travaux menés par le tribun Titus Suedius Clemens et a surtout servi à identifier un mur antérieur dont une partie du tracé différait. Cela autorise à repenser les actions de restitutio menées par ce tribun et à illustrer le fait que d'importants remaniements ont effectivement eu lieu dans l'espace périurbain de Pompéi. En effet, sous les niveaux éruptifs, un fort pendage vers l'est et le sud-est a pu être identifié et aucune structure funéraire n'a été identifiée. Cela laisse penser, au moins dans les derniers temps de la cité, que l'enclos ENC40001 présent à l'est de la voie partant vers le nord ne possédait probablement pas une vocation funéraire, comme cela pouvait être supposé. Une hypothèse serait de rattacher cet espace à des loca publica, faisant peut-être partie de ceux mentionnés par l'inscription du tribun Titus Suedius Clemens. Cette interprétation repose notamment sur les premières données stratigraphiques, illustrant la contemporanéité d'installation de la stèle inscrite SB10008 et du mur occidental MR40001 de l'enclos. La fonction propre de cet enclos reste imprécise au vu de la fenêtre d'étude et de l'impossibilité de l'agrandir, en raison de la présence du chemin actuel d'accès au site. Il s'agira d'ouvrir un secteur de fouille au sein de l'enclos ENC30001, de l'autre côté de la voie dans la zone 3, afin de tenter d'en savoir plus sur la fonction de ces vastes enclos et si l'aspect funéraire de ceux-ci peut être écarté. Une piste de recherche est apportée par un enclos du même type, délimité par un muret, dégagé au sud-est de Pompéi, au pied des remparts et directement à l'ouest de la porte de Nocera. Coincé entre les fortifications et la voie dite "pomériale", un espace semblable est en effet installé dans le prolongement d'une construction identifiée comme des écuries. Ces espaces pourraient donc être liés à des zones de pâturage et/ou de parcage, sous contrôle de la cité.

Enfin, les prochaines campagnes seront notamment consacrées à la poursuite des investigations sur le secteur 20, afin d'atteindre les niveaux de fondation des monuments funéraires MSL20003 et MSL20004 et d'en préciser la chronologie. De plus, il s'agira de mieux cerner le croisement des voies en avant de la porte de la ville, de reprendre l'étude de l'ensemble des monuments funéraires et de l'aqueduc, afin de compléter les connaissances de cette zone périurbaine autorisant à observer l'organisation et la gestion d'un secteur où s'articulent des structures défensives, 
viaires, hydrauliques, funéraires, résidentielles, artisanales et des décharges installées sur des terrains publics ou privés.

\section{BIBLIOGRAPHIE}

CAMPBELL 2015

Virginia L. Campbell, The Tombs of Pompeii: Organization, Space, and Society, New York - Londres, Routledge, 2015.

DELLA CORTE 1913

Matteo Della Corte, « Il pomerium di Pompei », Rendiconti della Reale Accademia dei Lincei, Rome, Reale Accademia dei Lincei, Classe di Scienze Morali, Storiche e Filologiche, s. 5 22, 1913,

p. 261-308.

GARCIA Y GARCIA 2006

Laurentino García y García, Danni di guerra a Pompei : una dolorosa vicenda quasi dimenticata, Studi della Soprintendenza archeologica di Pompei 15, 2006.

JIMÉNEZ DE FURUNDARENA 1999

Augustín Jiménez de Furundarena, « La carrera de Titus Suedius Clemens », Hispania antiqua 23, 1999, p. 167-172.

LEMAIRE 2017

Bastien Lemaire, Aux marges de la ville antique en Méditerranée occidentale : de l'urbain au périurbain. De l'approche diachronique à l'étude de cas campaniens : Cumes et Pompéi, Doctorat d'Archéologie de la Méditerranée Antique, Montpellier, 2017.

LEMAIRE, BRUN 2020

Bastien Lemaire, Jean-Pierre Brun, « Du Fondo Barbatelli à la Porte du Vésuve : Une fenêtre d'étude dans le faubourg septentrional de Pompéi. Campagne 2019. ", Chronique des activités archéologiques de l'École française de Rome, en ligne, URL : http://journals.openedition.org/cefr/4941, consulté le 24 janvier 2021.

MOLS, MOORMAN 1993

Stephan Mols, Eric M. Moormann, « Ex parvo crevit. Proposta per una lettura iconografica della Tomba di Vestorius Priscus fuori Porta Vesuvio a Pompei », Rivista di Studi Pompeiani 6, 1993, p. 15-52.

SEILER et al. 2005

Florian Seiler, Heinz Beste, Carla Piraino, Domenico Esposito, « La Regio VI e la zona della porta Vesuvio ", Nuove ricerche archeologiche a Pompei ed Ercolano, Studi della Soprintendenza archeologica di Pompei 10, Naples, 2005, p. 216-234.

SPANO 1910

Giovanni Spano, « Pompei. Relazione degli scavi eseguiti negli anni 1908 e 1909 », Notizie degli scavi di Antichità, 1910, p. 377-418. 
STEFANI 1995-1996

Grete Stefani, «Contributo alla carta archeologica dell' “ager pompeianus”. I rinvenimenti presso

Porta Vesuvio », Rivista di Studi Pompeiani 7 (1995-1996), p. 11-33.

STEFANILE 2016

Michele Stefanile, «Ceius Secundus, Helvius Sabinus, Suedius Clemens : alcune novità epigrafiche dalla schedatura dei tituli picti pompeiani per EDR », Vesuviana 8, p. 85-104.

OHLIG 2001

Christoph P. J. Ohlig, De Aquis Pompeiorum. Das Castellum Aquae in Pompeji : Herkunft, Zuleitung und Verteilung des Wassers, Nijmegen, C. Ohlig, 2001.

\section{NOTES}

1. Voir notamment SEILER et al. 2005, p. 216-234.

2. Pour un premier point sur cette zone suburbaine, voir LEMAIRE, BRUN 2020.

3. STEFANI 1995-1996; LEMAIRE 2017.

4. OHLIG 2001.

5. Voir notamment JIMÉNEZ DE FURUNDARENA 1999 et en dernier lieu STEFANILE 2016.

6. Cette stèle a été dégagée lors de la campagne 2019 : voir LEMAIRE, BRUN 2020.

7. «Ex auctoritate / imp(eratoris) Caesaris / Vespasiani Aug(usti) / loca publica / a privatis possessa T(itus) Suedius /Clemens tribunus causis cognitis / et mensuris factis rei publicae | Pompeianorum restituit»: «En vertu de l'autorité de l'empereur César Vespasien Auguste, le tribun Titus Suedius Clemens, après avoir instruit l'affaire et effectué des mesures, a restitué à la Res Publica des pompéiens les lieux publics occupés par des particuliers $»$.

8. DELLA CORTE 1913, p. 261.

9. SPANO 1910, p. 409-416.

10. Titus Suedius Clemens est par la suite envoyé en Égypte ; voir notamment JIMÉNEZ DE FURUNDARENA 1999 et STEFANILE 2016.

11. SPANO 1910, fig. 12, p. 401, MOLS, MOORMAN 1993, fig. 1.

12. LEMAIRE, BRUN 2020.

13. Voir notamment S PANO 1910, p.399-416, sur les découvertes réalisées lors des dégagements.

14. CAMPBELL 2015, p. 203-204.

15. Epigraphic Database Roma online ( http://www.edr-edr.it): Schedae numerus EDR143590 (U. Soldovieri).

16. CAMPBELL 2015, p. 204-205.

17. Epigraphic Database Roma online (http://www.edr-edr.it): Schedae numerus EDR072570 (U. Soldovieri).

18. LEMAIRE, BRUN 2020.

19. Voir SPANO 1910, p. 399-416, sur les découvertes réalisées lors des dégagements et notamment lors de la fouille de ce vaste dépotoir. 
20. GARCIA Y GARCIA 2006.

21. Ce retour n'a pu être identifié lors de nos investigations, mais est visible sur d'anciennes photographies (par exemple sur un cliché aérien de 1910: STEFANI 1995-1996, p. 22, fig. 9).

22. STEFANI 1995-1996, fig. 9, p. 22.

INDEX

Thèmes : $\mathrm{CJB}$

Année de l'opération : 2020

lieux https://ark.frantiq.fr/ark:/26678/pcrt7ya9w00Xhb

chronologie https://ark.frantiq.fr/ark:/26678/pcrtZTmusVUU24

peuples https://ark.frantiq.fr/ark:/26678/pcrtkERiWybjc4

sujets https://ark.frantiq.fr/ark:/26678/pcrtJfGF3Plikr, https://ark.frantiq.fr/ark:/26678/

pcrtjclbYvph1S, https://ark.frantiq.fr/ark:/26678/pcrteJiI6BxaFN, https://ark.frantiq.fr/ark:/

26678/pcrtsIm3RuNMGu

\section{AUTEURS}

\section{BASTIEN LEMAIRE}

UMR 5140, Archéologie des sociétés méditerranéennes - Université Paul Valéry Montpellier 3

\section{JEAN-PIERRE BRUN}

Collège de France 